\title{
3. (1) Burden and centralised treatment in Europe of rare tumours: results of RARECAREnet-a population-based study
}

\author{
Gemma Gatta, Riccardo Capocaccia, Laura Botta, Sandra Mallone, Roberta De Angelis, Eva Ardanaz, Harry Comber, Nadya Dimitrova, \\ Maarit K Leinonen, Sabine Siesling, Jan M van der Zwan, Liesbet Van Eycken, Otto Visser, Maja P Žakelj, Lesley A Anderson, Francesca Bella, \\ Kaire Innos, Renée Otter, Charles A Stiller, Annalisa Trama, for the RARECAREnet working group*
}

\section{Summary}

Lancet Oncol 2017; 18: 1022-39

Published Online

July 4, 2017

http://dx.doi.org/10.1016/

S1470-2045(17)30445-X

This online publication has been corrected. The corrected version first appeared at thelancet.com/oncology on July 26, 2017

See Comment page 983

${ }^{*}$ Members of the RARECARENet Working Group are listed in the appendix

Evaluative Epidemiology Unit, Fondazione IRCCS, Istituto Nazionale dei Tumori, Milan, Italy $(\mathrm{G}$ Gatta $\mathrm{MD}$, R Capocaccia MSc, L Botta MSc, A Trama PhD); Centro Nazionale Prevenzione delle malattie $\mathrm{e}$ Promozione della Salute (CNAPPS) Istituto Superiore di Sanità, Rome, Italy (S Mallone MSc); Dipartimento di Oncologia e Medicina Molecolare, Istituto Superiore

di Sanità, Rome, Italy (R De Angelis MSc); CIBER de Epidemiología y Salud Pública (CIBERESP), Spain (Eva Ardanaz PhD); Instituto de Salud Pública de Navarra, Pamplona, Spain (Eva Ardanaz);

National Cancer Registry Ireland, Cork, Ireland (H Comber PhD); Bulgarian National Cancer Registry, Sofia, Bulgaria (N Dimitrova PhD); Cancer Society of Finland, Finnish Cancer Registry, Helsinki, Finland (M K Leinonen PhD); Department of Research, Netherlands Comprehensive Cancer Organisation (IKNL), Utrecht, the Netherlands ( $\mathrm{S}$ Siesling PhD,

J M van der Zwan PhD); Belgian Cancer Registry, Brussels, Belgium (L Van Eycken MD); Netherlands Comprehensive Cancer Organisation (IKNL), Utrecht, the Netherlands (O Visser PhD); Institute of Oncology Ljubljana, Epidemiology and Cancer Registry, Ljubljana, Slovenia

Background Rare cancers pose challenges for diagnosis, treatments, and clinical decision making. Information about rare cancers is scant. The RARECARE project defined rare cancers as those with an annual incidence of less than six per 100000 people in European Union (EU). We updated the estimates of the burden of rare cancers in Europe, their time trends in incidence and survival, and provide information about centralisation of treatments in seven European countries.

Methods We analysed data from 94 cancer registries for more than 2 million rare cancer diagnoses, to estimate European incidence and survival in 2000-07 and the corresponding time trends during 1995-2007. Incidence was calculated as the number of new cases divided by the corresponding total person-years in the population. 5-year relative survival was calculated by the Ederer-2 method. Seven registries (Belgium, Bulgaria, Finland, Ireland, the Netherlands, Slovenia, and the Navarra region in Spain) provided additional data for hospitals treating about 220000 cases diagnosed in 2000-07. We also calculated hospital volume admission as the number of treatments provided by each hospital rare cancer group sharing the same referral pattern.

Findings Rare cancers accounted for $24 \%$ of all cancers diagnosed in the EU during 2000-07. The overall incidence rose annually by $0.5 \%(99 \cdot 8 \%$ CI $0 \cdot 3-0 \cdot 8)$. 5 -year relative survival for all rare cancers was $48 \cdot 5 \%(95 \%$ CI $48 \cdot 4$ to $48 \cdot 6)$, compared with $63.4 \%(95 \%$ CI 63.3 to 63.4$)$ for all common cancers. 5-year relative survival increased (overall $2.9 \%$, 95\% CI $2 \cdot 7$ to $3 \cdot 2$ ), from 1999-2001 to 2007-09, and for most rare cancers, with the largest increases for haematological tumours and sarcomas. The amount of centralisation of rare cancer treatment varied widely between cancers and between countries. The Netherlands and Slovenia had the highest treatment volumes.

Interpretation Our study benefits from the largest pool of population-based registries to estimate incidence and survival of about 200 rare cancers. Incidence trends can be explained by changes in known risk factors, improved diagnosis, and registration problems. Survival could be improved by early diagnosis, new treatments, and improved case management. The centralisation of treatment could be improved in the seven European countries we studied.

Funding The European Commission (Chafea).

\section{Introduction}

The RARECARE project ${ }^{1}$ defined rare cancers as those with an annual incidence of less than six per 100000 people in the European Union (EU), and showed that about one in five cancers diagnosed in the EU were rare and slightly more than 4 million people with a rare cancer diagnosis were living in the EU population. Because of their low numbers, the almost 200 rare cancers listed by RARECARE pose challenges for diagnosis, treatments, and clinical decision making Clinical trials into such cancers are rare too, and it is hard to build up new knowledge and expertise.

The broad consensus suggests that diagnostic pathological confirmation and primary treatment of rare cancers, in particular, should be centralised in reference centres, collaborative networks, or both, with multidisciplinary approaches ${ }^{2}$ and very specific expertise. Additionally, clinical and translational research calls for a high level of centralisation and international collaboration. To what extent appropriate policies for patients with rare cancer are implemented at the country level has seldom been studied. As a consequence, information for policy makers and stakeholders is scarce for many of these rare tumours.

The project Information Network on Rare Cancers (RARECAREnet) is designed to update epidemiological information about rare cancers in the $\mathrm{EU},{ }^{3}$ to provide indicators at the country level, time trends, and to study to what extent treatment is centralised in Europe.

In this study, we provide current incidence and survival estimates based on data collected from 94 populationbased cancer registries, for 198 rare cancers diagnosed in 2000-07 and for 12 major families of rare cancers. We also present data for the levels of centralisation for rare cancers in selected European countries.

\section{Methods}

\section{Patients}

We extracted data from two databases. The first, the descriptive analysis database, is a subset of the EUROCARE-5 database, ${ }^{4}$ which includes incidence and follow-up data provided by European population-based 
Research in context

\section{Evidence before the study}

The surveillance of rare cancer in Europe (RARECARE) project provided data from European population-based cancer registries to provide for the first time the burden of rare cancers in Europe. The RARECARE definition of rare cancer was an incidence of less than six cases per 100000 people per year. A list of rare cancers was created, combining the topography and morphology codes from the International Classification of Diseases for Oncology (ICD-0-3). The list was produced after consultations by a group of pathologists, clinicians and epidemiologists, and was endorsed by the main European cancer organisations. The definition and the list were employed in several European and extra-European countries. The European Commission (EC) based the recent launch of the Joint Action on Rare Cancer in line with this definition. Estimates indicated that about one in five new cancers were rare and slightly more than 4 million people diagnosed with rare cancers lived in Europe. Outcomes (5-year relative survival) were worse for patients with rare cancers than for patients with common cancers.

There is general agreement that treatment of rare cancers should be concentrated in specialised multidisciplinary centres, and that international collaboration is needed for research on these cancers. However, there is no knowledge about the extent of centralisation of rare cancer treatment at the population level.

\section{Added value of this study}

With the new project 'Information network on rare cancer' (RARECAREnet), we updated the burden of rare cancer and provided indicators of the centralisation of patients with rare cancer in seven European countries. We estimated about 650000 new diagnoses of rare cancers occur yearly in Europe, with an incidence of 115 of 100000 per year. The incidence rose by $0.5 \%$ annually, due to overdiagnosis (eg, thyroid carcinoma) or improved diagnosis (eg, neuroendocrine tumours [NET], gastrointestinal stromal tumour [GIST]) or increases in exposure to risk factors (such as HPV). 5-year survival for rare cancers (49\%) is still lower than for common cancers (63\%), but has risen, from $46 \%$ in $1999-2001$ to $49 \%$ in $2005-07$. Significant progress was reported for some poor-prognosis cancers, such as chronic myeloid leukaemia, gastroenteropancreatic tumours (GEP), soft tissue sarcoma of viscera, plasmocytoma or multiple myeloma, and oesophageal cancers. Seven European countries provided data to study the extent of hospital centralisation for rare cancers and these data showed that, overall, centralisation of rare cancer treatment varied widely between countries and was generally low.

\section{Implication of all the evidence}

Rare cancers are a specific group of rare diseases requiring, compared with other rare diseases, the largest expenditure for drugs. The rare disease community has strongly lobbied the EC to increase investment in research, management, and social support for these diseases. This very large population-based study on rare cancers thus provides useful information for public health. The EC recognised the results of RARECARE and RARECAREnet and launched a European Joint Action for Rare Cancers in 2016 aiming to help member states with their national health plans. The $\mathrm{EC}$ also recently approved two specific rare cancer European Reference Networks (ERN), for childhood and solid cancers, to exchange management of very rare cancers or to treat patients from small countries. Both initiatives can use these data, with national and European health-care institutions, to address the issue of rare cancers better.

Lower survival of patients with rare cancer might be explained by difficulties in clinical research, as well as their specific biological characteristics, and our results have shown that non-optimal organisation of care can have a role too. There is ample room for improving the levels of centralisation of treatment and clinical expertise in seven European countries. These results were discussed in national meetings, and confirmed the need for improving the centralisation of rare cancer treatment in fewer, more specialised hospitals. Our data offer a starting point to measure the effects of new policies on rare cancers.

Data provided by cancer registries are essential, but their quality has to improve, especially on morphology, hospital and treatment definitions. Basic information on stage and the determinants of stage needs to be collected routinely. The European network of cancer registries should work to boost these improvements and make wider use of the data on rare cancers.
(M P Žakelj DSc); Centre for Public Health, School of Medicine, Dentistry and Biomedical Science, Queen's University Belfast, Belfast, UK (LA Anderson PhD); Integrated Cancer Registry of

Catania-Messina-Siracusa-Enna, Azienda

Ospedaliero-Universitaria Policlinico-Vittorio Emanuale Catania, Italy (F Bella MD) Department of Epidemiology and Biostatistics, National Institute for Health Development, Tallinn, Estonia (K Innos PhD); Belgian Cancer Center, Department of Public Health and

Surveillance, Scientific Institute of Public Health, Brussels, Belgium (R Otter PhD); and National Cancer Registration and Analysis Service, Public Health England, Oxford, UK (C A Stiller MSc)

Correspondence to: Gemma Gatta, Evaluative Epidemiology Unit, Fondazione IRCCS, Istituto Nazionale dei Tumori, Milan 20133, Italy gemma.gatta@istitutotumori. mi.it cancer registries for patients with cancer diagnosed between Jan 1, 1978, and Dec 31, 2007. Vital status was updated to Dec 31, 2008. From the 117 cancer registries participating in EUROCARE-5, we excluded specialised paediatric cancer registries, the Swedish and Turin cancer registries, because they did not participate in the RARECAREnet study, and the Danish cancer registry, because it did not provide the morphology detail needed to define rare cancers. Details of the RARECAREnet database can be found in the report on the project website. ${ }^{5}$ To analyse incidence, we excluded 11 anatomicalsite-specific cancer registries to avoid incomplete coverage of some cancer entities affecting multiple sites such as neuroendocrine tumours. We excluded cases identified only by death certificate or incidentally discovered at autopsy from the analysis because they do not report time of survival. Cases lost to follow-up were considered as censored at the date of last contact. We included multiple primaries in the same patient.

The second database was used for the study of hospitals of treatment and hospital volume, which includes data from seven European cancer registries: the national cancer registries of Belgium, Bulgaria, Finland, Ireland, the Netherlands, Slovenia, and the regional cancer 
registry of Navarra (Spain). The Navarra cancer registry, although regional, was added because of the regional organisation of the Spanish health-care system. These cancer registries were selected to reflect the variability of incidence and survival in Europe, ${ }^{1,5}$ and because they could provide detailed data for all 198 rare cancers included in the RARECAREnet list. Variables included in the database were: sex, date of birth, date of diagnosis, topography and morphology codes from the International Classification of Disease for Oncology version 3 (ICDO-3) grading, pathological and clinical TNM Classification of Malignant Tumours (TNM) staging, simplified stage (localised, regional extension, metastatic), treatment (surgery, radiotherapy, systemic, other, or none), vital status, date of last follow-up or death, hospital of diagnosis, and hospital of treatment. The hospital of diagnosis was defined as the hospital where the pathology examination was done or requested. The hospital of treatment(s) was defined as the hospital where a specific treatment (eg, surgery) or the first course of systemic therapy (eg, chemotherapy) was given. Up to five different types of treatment within 1 year from the date of diagnosis were considered as a primary treatment. Vital status was further updated in this second database, with respect to the first descriptive analysis database, to Dec 31, 2012.

\section{Analysis}

The rationale for the definition of rare cancer entities and their classification in terms of ICD-O codes are reported elsewhere. ${ }^{1,2,5}$ Classification was structured to avoid any overlap among rare entities. For example, gastroenterohepatic neuroendocrine tumours and gastrointestinal stromal tumours were under the families of neuroendocrine tumours and sarcomas, but not also in digestive rare cancers.

From the first database, we estimated incidence as the number of new cases arising in 2000-07 divided by the corresponding total person-years (male and female) in the general population. The European standard population was used for direct age standardisation. New cases in 2013 in EU28 (28 member states of EU) were calculated by multiplying age-specific and sex-specific incidence rates in 2000-07 by the corresponding European population classified in 5-year age classes on Jan 1, 2013.

We estimated incidence variation by restricting the analysis to cases diagnosed in the two sub-periods 1999-2002 and 2003-07, and presented the results in a funnel plot. Annual percentage change was calculated as the ratio between incidence rates for the two sub-periods to the power of 1 over $4 \cdot 5$, the inverse of the mean difference in time between the two sub-periods.

We estimated 5-year relative survival as the ratio of observed to expected survival in the general population, matched by age, sex, calendar year, and geographical area, and calculated by the Ederer- 2 method. ${ }^{6}$ We estimated relative survival time trends using the period approach and considering three follow-up periods: 1999-2001 (cohorts diagnosed in Jan 1, 1995, to Dec 31, 2001), 2002-04 (cohorts diagnosed in Jan 1,1998 to Dec 31, 2004), and 2005-07 (cohorts diagnosed in Jan 1, 2001, to Dec 31, 2007). We presented relative survival changes as a funnel plot.

The volume (number) of treatments provided by each hospital was calculated from the second database for major cancer groups, defined by aggregating all the solid rare cancers into 38 groups sharing the same referral pattern. For example, all seven head and neck tumours identified as clinically distinct rare entities ${ }^{1}$ are usually referred to head and neck specialised services, and we considered these as a single group. Haematological rare tumours do not always require hospitalisation and we did not consider these in the volume analysis. We then computed hospital volume for each of the 38 groups as the annual number of any treatment delivered by the hospital, for all the cancers in that group. We regarded repeated admissions to the same hospital for the same cancer and the same treatment type (ie, surgery, radiotherapy, or systemic therapy) as a single admission and counted as one treatment in the analyses. Repeated admissions for several treatment types (such as radiotherapy and subsequent surgery) given to a patient in the same hospital were counted as separate treatments. Untreated patients were assigned to the hospital of diagnosis. The total number of treatments provided by each hospital for a given group of rare cancers was then divided by the number of years of observation to provide its mean annual hospital volume. Pearson's correlation coefficient was used to evaluate the association, across cancers, between mean admission volume and incidence.

Finally, for each patient we calculated the mean annual volume of the hospital(s) where they were treated to obtain a patient-specific measure with a much less skewed distribution with respect to the hospital-specific volume. The mean value of this measure for all the patients diagnosed with a given group of rare cancers in a certain country gives a cancer-specific and countryspecific measure of the level of expertise that patients can expect for the treatment of their tumour. We called this calculation the mean admission volume indicator.

\section{Role of the funding source}

The funders had no role in study design, collection, analysis or interpretation of data, or writing of the report. The corresponding author had full access to all the data and had final responsibility for the decision to submit for publication.

\section{Results}

Survival estimates in 2000-07 for all rare cancers were based on 1994346 diagnoses, observed by 94 cancer registries. 1984147 rare cancer diagnoses were considered for incidence estimates in $2000-07$, collected by 83 cancer registries from 1566 million person-years of observation. 
62828 (3\%) of 1984147 cases were identified by death certificate only or incidentally at autopsy ranging from a maximum of $4412(10 \%)$ of 44755 cases in Slovakia, to $265(<1 \%)$ of 60377 cases in Scotland. Data for incidence time trends came from 42 cancer registries for 1995-2007, and included 2268602 cases, and 1900 million personyears of observation. Survival time trend analysis was based on 1649309 rare cancer diagnoses from 45 cancer registries providing uninterrupted data from at least Jan 1, 1995, to Dec 31, 2007.

For the study of hospitals of treatment and hospital volume, we received data for about 348000 rare cancers diagnosed in the period 2000-07. However, national data from Belgium were limited to 2004-07, and those from Navarra to 2000-05. Cases diagnosed in Bulgaria and the Netherlands during 2000-04 were removed on account of incomplete national coverage of hospital information. 223081 rare cancer cases were included in the hospital volume study database. Non-specific morphologies $(8000,8001,8010,8800,9800,9590)$ were found in 4588 (2\%), with the highest proportion in Finland (1268 [4\%] of 30740 cases). 37959 (17\%) of 223081 cases were removed because hospital information was missing.

Table 1 shows the incidence and survival estimates for each of the 198 rare cancers, for 63 groups of rare cancers, for the 12 wider families in which rare cancers are hierarchically grouped, and for six common cancer groups. Haematological malignancies, rare cancers of female genital organs and of the digestive tract, and head and neck cancers were families with the highest overall incidence rates (from 19 to 28 per 100000 people per year). Thoracic cancers, male

\begin{tabular}{|c|c|c|c|c|c|c|c|}
\hline & $\begin{array}{l}\text { Crude incidence } \\
\text { rate per } \\
100000 \text { people } \\
\text { per year }\end{array}$ & $95 \% \mathrm{Cl}$ & $\begin{array}{l}\text { Observed cases } \\
\text { in } 83 \text { cancer } \\
\text { registries in } \\
2000-07\end{array}$ & $\begin{array}{l}\text { Estimated } \\
\text { new cases in } \\
2013 \text { in EU28 }\end{array}$ & $\begin{array}{l}5 \text {-year } \\
\text { relative } \\
\text { survival (\%) }\end{array}$ & $95 \% \mathrm{Cl}$ & $\begin{array}{l}\text { Observed cases } \\
\text { in } 94 \text { cancer } \\
\text { registries in } \\
2000-07\end{array}$ \\
\hline Rare: head and neck cancers & $18 \cdot 82$ & $16 \cdot 76-16 \cdot 89$ & 263565 & 84989 & $52 \cdot 1 \%$ & $51 \cdot 8-52 \cdot 3$ & 254563 \\
\hline Epithelial tumours of nasal cavity and sinuses & 0.45 & $0.44-0.46$ & 7046 & 2282 & $47 \cdot 3 \%$ & $45 \cdot 8-48 \cdot 8$ & 6867 \\
\hline $\begin{array}{l}\text { Squamous cell carcinoma with variants of nasal cavity and } \\
\text { sinuses }\end{array}$ & $0 \cdot 35$ & $0 \cdot 34-0 \cdot 36$ & 5465 & 1770 & $49 \cdot 5 \%$ & $47 \cdot 8-51 \cdot 2$ & 5444 \\
\hline Lymphoepithelial carcinoma of nasal cavity and sinuses & 0.00 & $0.00-0.00$ & 31 & 10 & $70 \cdot 8 \%$ & $50 \cdot 7-99 \cdot 0$ & 31 \\
\hline Undifferentiated carcinoma of nasal cavity and sinuses & 0.02 & $0.02-0.02$ & 286 & 93 & $30 \cdot 5 \%$ & $24 \cdot 3-38 \cdot 2$ & 283 \\
\hline Intestinal-type adenocarcinoma of nasal cavity and sinuses & 0.00 & $0.00-0.00$ & 42 & 14 & $65 \cdot 0 \%$ & $48 \cdot 9-86 \cdot 4$ & 42 \\
\hline Epithelial tumours of nasopharynx & 0.47 & $0.46-0.49$ & 7439 & 2580 & $48.9 \%$ & $47 \cdot 5-50 \cdot 2$ & 7276 \\
\hline Squamous cell carcinoma with variants of nasopharynx & 0.36 & $0 \cdot 35-0.37$ & 5613 & 1941 & $48 \cdot 5 \%$ & $47 \cdot 0-50 \cdot 1$ & 5589 \\
\hline Papillary adenocarcinoma of nasopharynx & 0.00 & $0.00-0.00$ & 17 & 6 & $58 \cdot 7 \%$ & $36 \cdot 2-95 \cdot 3$ & 17 \\
\hline $\begin{array}{l}\text { Epithelial tumours of major salivary glands and salivary-gland- } \\
\text { type tumours }\end{array}$ & 1.39 & $1 \cdot 37-1 \cdot 41$ & 21794 & 7059 & $62 \cdot 8 \%$ & $62 \cdot 0-63 \cdot 7$ & 21364 \\
\hline Epithelial tumours of major salivary glands & 0.96 & $0.95-0.98$ & 15053 & 4876 & $60 \cdot 8 \%$ & $59 \cdot 8-61 \cdot 8$ & 14703 \\
\hline Salivary-gland-type tumours of head and neck & 0.43 & $0.42-0.44$ & 6741 & 2183 & $67 \cdot 1 \%$ & $65 \cdot 7-68 \cdot 6$ & 6683 \\
\hline Epithelial tumours of hypopharynx and larynx & $6 \cdot 33$ & $6 \cdot 29-6 \cdot 37$ & 99176 & 31545 & $52 \cdot 0 \%$ & $51 \cdot 6-52 \cdot 4$ & 96793 \\
\hline Squamous cell carcinoma with variants of hypopharynx & $1 \cdot 27$ & $1.25-1.28$ & 19828 & 6422 & $25 \cdot 1 \%$ & $24 \cdot 4-25 \cdot 9$ & 19878 \\
\hline Squamous cell carcinoma with variants of larynx & $4 \cdot 61$ & $4 \cdot 58-4 \cdot 64$ & 72210 & 23389 & $60 \cdot 5 \%$ & $60 \cdot 1-61 \cdot 0$ & 71928 \\
\hline Epithelial tumours of oropharynx & $3 \cdot 32$ & $3 \cdot 29-3 \cdot 35$ & 52017 & 16848 & $40 \cdot 9 \%$ & $40 \cdot 4-41 \cdot 4$ & 50843 \\
\hline Squamous cell carcinoma with variants of oropharynx & $3 \cdot 12$ & $3 \cdot 09-3 \cdot 14$ & 48812 & 15810 & $41 \cdot 3 \%$ & $40 \cdot 8-41 \cdot 8$ & 48401 \\
\hline Epithelial tumours of oral cavity and lip & $4 \cdot 78$ & $4 \cdot 75-4 \cdot 81$ & 74890 & 24257 & $56 \cdot 7 \%$ & $56 \cdot 2-57 \cdot 1$ & 73101 \\
\hline Squamous cell carcinoma with variants of oral cavity & $3 \cdot 51$ & $3 \cdot 48-3 \cdot 54$ & 54931 & 17792 & $48.0 \%$ & $47 \cdot 5-48 \cdot 6$ & 54229 \\
\hline Squamous cell carcinoma with variants of lip & 1.02 & $1.00-1.04$ & 15984 & 5177 & $89 \cdot 5 \%$ & $88 \cdot 5-90 \cdot 5$ & 15899 \\
\hline Epithelial tumours of eye and adnexa & 0.04 & $0.04-0.05$ & 679 & 247 & $80 \cdot 6 \%$ & $75 \cdot 9-85 \cdot 6$ & 673 \\
\hline Squamous cell carcinoma with variants of eye and adnexa & 0.03 & $0.02-0.03$ & 421 & 136 & $88 \cdot 9 \%$ & $83 \cdot 0-95 \cdot 2$ & 422 \\
\hline Adenocarcinoma with variants of eye and adnexa & 0.01 & $0 \cdot 01-0 \cdot 01$ & 134 & 43 & $58 \cdot 7 \%$ & $49 \cdot 1-70 \cdot 1$ & 134 \\
\hline Epithelial tumours of middle ear & 0.03 & $0.03-0.04$ & 524 & 170 & $44 \cdot 1 \%$ & $38 \cdot 5-49 \cdot 6$ & 488 \\
\hline Squamous cell carcinoma with variants of middle ear & 0.02 & $0.02-0.03$ & 377 & 122 & $37 \cdot 6 \%$ & $31 \cdot 8-44 \cdot 4$ & 370 \\
\hline Adenocarcinoma with variants of middle ear & 0.00 & $0.00-0.00$ & 50 & 16 & $83 \cdot 8 \%$ & $70 \cdot 5-99 \cdot 5$ & 50 \\
\hline Rare: digestive cancers & 21.94 & $21 \cdot 86-22 \cdot 01$ & 343635 & 112351 & $15 \cdot 3 \%$ & $15 \cdot 2-15 \cdot 5$ & 321375 \\
\hline Epithelial tumours of oesophagus & $7 \cdot 81$ & $7 \cdot 77-7 \cdot 85$ & 122344 & 40068 & $12 \cdot 0 \%$ & $11 \cdot 8-12 \cdot 2$ & 119522 \\
\hline Squamous cell carcinoma with variants of oesophagus & $3 \cdot 36$ & $3 \cdot 33-3 \cdot 39$ & 52597 & 17036 & $11 \cdot 7 \%$ & $11 \cdot 3-12 \cdot 0$ & 53225 \\
\hline Adenocarcinoma with variants of oesophagus & $3 \cdot 26$ & $3 \cdot 24-3 \cdot 29$ & 51138 & 16564 & $13 \cdot 9 \%$ & $13 \cdot 5-14 \cdot 2$ & 51250 \\
\hline Salivary-gland-type tumours of oesophagus & 0.00 & $0.00-0.01$ & 63 & 20 & $13 \cdot 7 \%$ & $6 \cdot 4-29 \cdot 0$ & 64 \\
\hline Undifferentiated carcinoma of oesophagus & 0.04 & $0.04-0.05$ & 695 & 225 & $6 \cdot 8 \%$ & $4 \cdot 9-9 \cdot 4$ & 712 \\
\hline
\end{tabular}




\begin{tabular}{|c|c|c|c|c|c|c|c|}
\hline & $\begin{array}{l}\text { Crude incidence } \\
\text { rate per } \\
100000 \text { people } \\
\text { per year }\end{array}$ & $95 \% \mathrm{Cl}$ & $\begin{array}{l}\text { Observed cases } \\
\text { in } 83 \text { cancer } \\
\text { registries in } \\
2000-07\end{array}$ & $\begin{array}{l}\text { Estimated } \\
\text { new cases in } \\
2013 \text { in EU28 }\end{array}$ & $\begin{array}{l}5 \text {-year } \\
\text { relative } \\
\text { survival (\%) }\end{array}$ & $95 \% \mathrm{Cl}$ & $\begin{array}{l}\text { Observed cases } \\
\text { in } 94 \text { cancer } \\
\text { registries in } \\
2000-07\end{array}$ \\
\hline \multicolumn{8}{|l|}{ (Continued from previous page) } \\
\hline Rare epithelial tumours of stomach & 0.33 & $0.32-0.34$ & 5146 & 1886 & $15 \cdot 9 \%$ & $14 \cdot 7-17 \cdot 1$ & 5157 \\
\hline Squamous cell carcinoma with variants of stomach & 0.12 & $0 \cdot 11-0 \cdot 12$ & 1807 & 585 & $17 \cdot 5 \%$ & $15 \cdot 6-19 \cdot 7$ & 1800 \\
\hline Salivary-gland-type tumours of stomach & 0.00 & $0.00-0.00$ & 39 & 13 & $25 \cdot 1 \%$ & $12 \cdot 7-49 \cdot 9$ & 40 \\
\hline Undifferentiated carcinoma of stomach & 0.21 & $0 \cdot 20-0 \cdot 22$ & 3300 & 1069 & $14 \cdot 9 \%$ & $13 \cdot 5-16 \cdot 4$ & 3317 \\
\hline Epithelial tumours of small intestine & 0.77 & $0.76-0.79$ & 12132 & 3930 & $27 \cdot 3 \%$ & $26 \cdot 3-28 \cdot 3$ & 11544 \\
\hline Adenocarcinoma with variants of small intestine & 0.59 & $0.58-0.60$ & 9219 & 2986 & $27 \cdot 9 \%$ & $26 \cdot 8-29 \cdot 0$ & 9193 \\
\hline Squamous cell carcinoma with variants of small intestine & 0.01 & $0.01-0.01$ & 133 & 43 & $34.8 \%$ & $26 \cdot 8-45 \cdot 3$ & 133 \\
\hline Rare epithelial tumour of colon & 0.13 & $0.13-0.14$ & 2074 & 737 & $54 \cdot 8 \%$ & $52 \cdot 0-57 \cdot 7$ & 2075 \\
\hline Squamous cell carcinoma with variants of colon & 0.03 & $0.02-0.03$ & 400 & 130 & $37 \cdot 1 \%$ & $31 \cdot 8-43 \cdot 4$ & 395 \\
\hline $\begin{array}{l}\text { Fibromixoma and low-grade mucinous adenocarcinoma of the } \\
\text { appendix }\end{array}$ & 0.11 & $0 \cdot 10-0 \cdot 11$ & 1674 & 542 & $58 \cdot 8 \%$ & $55 \cdot 7-62 \cdot 1$ & 1680 \\
\hline Rare epithelial tumours of rectum & 0.11 & $0.11-0.12$ & 1764 & 635 & $47 \cdot 2 \%$ & $44 \cdot 4-50 \cdot 2$ & 1777 \\
\hline Squamous cell carcinoma with variants of rectum & 0.11 & $0.11-0.12$ & 1764 & 571 & $47 \cdot 2 \%$ & $44 \cdot 4-50 \cdot 2$ & 1777 \\
\hline Epithelial tumours of anal canal & $1 \cdot 16$ & $1 \cdot 14-1 \cdot 18$ & 18155 & 5880 & $56.5 \%$ & $55 \cdot 5-57 \cdot 4$ & 18020 \\
\hline Squamous cell carcinoma with variants of anal canal & 0.81 & $0.80-0.82$ & 12691 & 4111 & $63.0 \%$ & $61 \cdot 9-64 \cdot 1$ & 12847 \\
\hline Adenocarcinoma with variants of anal canal & 0.25 & $0.25-0.26$ & 3970 & 1286 & $41 \cdot 9 \%$ & $39 \cdot 9-43 \cdot 9$ & 3945 \\
\hline Paget's disease of anal canal & 0.00 & $0.00-0.00$ & 21 & 7 & $62.9 \%$ & $38 \cdot 0-104 \cdot 0$ & 21 \\
\hline Rare epithelial tumours of pancreas & 0.07 & $0.07-0.08$ & 1159 & 414 & $20 \cdot 2 \%$ & $17 \cdot 4-23 \cdot 3$ & 1116 \\
\hline Squamous cell carcinoma with variants of pancreas & 0.02 & $0.02-0.03$ & 361 & 117 & $5.9 \%$ & $3 \cdot 6-9 \cdot 6$ & 347 \\
\hline Acinar cell carcinoma of pancreas & 0.03 & $0.03-0.03$ & 449 & 145 & $19.0 \%$ & $14 \cdot 8-24 \cdot 3$ & 427 \\
\hline Mucinous cystadenocarcinoma of pancreas & 0.01 & $0.01-0.01$ & 109 & 35 & $35.9 \%$ & $26 \cdot 3-49 \cdot 0$ & 106 \\
\hline Intraductal papillary mucinous carcinoma invasive of pancreas & 0.01 & $0.01-0.01$ & 173 & 56 & $31.8 \%$ & $23 \cdot 6-42 \cdot 9$ & 171 \\
\hline Solid pseudopapillary carcinoma of pancreas & 0.00 & $0.00-0.00$ & 44 & 14 & $67 \cdot 7 \%$ & $52 \cdot 8-86 \cdot 8$ & 42 \\
\hline Serous cystadenocarcinoma of pancreas & 0.00 & $0.00-0.00$ & 4 & 1 & NE & NE & 4 \\
\hline Carcinoma with osteoclast-like giant cells of pancreas & 0.00 & $0.00-0.00$ & 19 & 6 & NE & $\mathrm{NE}$ & 19 \\
\hline Epithelial tumours of liver and intrahepatic bile tract & $7 \cdot 10$ & $7 \cdot 06-7 \cdot 14$ & 111271 & 36261 & $10 \cdot 1 \%$ & $9 \cdot 9-10 \cdot 3$ & 98765 \\
\hline Hepatocellular carcinoma of liver and intrahepatic bile tract & $3 \cdot 22$ & $3 \cdot 19-3 \cdot 25$ & 50461 & 16344 & $14.0 \%$ & $13 \cdot 7-14 \cdot 4$ & 46896 \\
\hline $\begin{array}{l}\text { Hepatocellular carcinoma, fibrolamellar of liver and } \\
\text { intrahepatic bile tract }\end{array}$ & 0.02 & $0.02-0.03$ & 387 & 125 & $28.1 \%$ & $23 \cdot 3-33 \cdot 8$ & 390 \\
\hline Cholangiocarcinoma of intrahepatic bile tract & 0.97 & $0.95-0.99$ & 15201 & 4924 & $6.0 \%$ & $5 \cdot 6-6 \cdot 6$ & 13845 \\
\hline $\begin{array}{l}\text { Adenocarcinoma with variants of liver and intrahepatic bile } \\
\text { tract }\end{array}$ & 0.41 & $0.40-0 \cdot 42$ & 6457 & 2091 & $6 \cdot 6 \%$ & $5 \cdot 9-7 \cdot 4$ & 6311 \\
\hline Undifferentiated carcinoma of liver and intrahepatic bile tract & 0.02 & $0.01-0.02$ & 240 & 78 & $2.7 \%$ & $1 \cdot 2-6 \cdot 4$ & 219 \\
\hline $\begin{array}{l}\text { Squamous cell carcinoma with variants of liver and } \\
\text { intrahepatic bile tract }\end{array}$ & 0.01 & $0.01-0.01$ & 147 & 48 & $14 \cdot 6 \%$ & $9 \cdot 1-23 \cdot 4$ & 143 \\
\hline Bile duct cystadenocarcinoma of intrahepatic bile tract & 0.00 & $0.00-0.00$ & 38 & 12 & $23 \cdot 6 \%$ & $11 \cdot 5-48 \cdot 5$ & 34 \\
\hline Epithelial tumours of gallbladder and extrahepatic biliary tract & $4 \cdot 44$ & $4 \cdot 41-4 \cdot 48$ & 69590 & 22540 & $13.6 \%$ & $13 \cdot 2-13 \cdot 9$ & 63889 \\
\hline Adenocarcinoma with variants of gallbladder & 1.35 & $1 \cdot 33-1 \cdot 36$ & 21085 & 6830 & $14 \cdot 5 \%$ & $14 \cdot 0-15 \cdot 1$ & 20338 \\
\hline Adenocarcinoma with variants of extrahepatic biliary tract & 1.44 & $1 \cdot 42-1 \cdot 46$ & 22510 & 7291 & $19 \cdot 2 \%$ & $18 \cdot 6-19 \cdot 8$ & 22234 \\
\hline $\begin{array}{l}\text { Squamous cell carcinoma of gallbladder and extrahepatic } \\
\text { biliary tract }\end{array}$ & 0.03 & $0.03-0.03$ & 496 & 161 & $8.8 \%$ & $6 \cdot 3-12 \cdot 3$ & 476 \\
\hline Rare: thoracic cancers & 6.80 & $6 \cdot 76-6 \cdot 84$ & 106573 & 37277 & $13 \cdot 4 \%$ & $13 \cdot 1-13 \cdot 6$ & 104670 \\
\hline Epithelial tumour of trachea & 0.11 & $0.11-0.12$ & 1771 & 574 & $18.0 \%$ & $16 \cdot 0-20 \cdot 3$ & 1697 \\
\hline Squamous cell carcinoma with variants of trachea & 0.06 & $0.06-0.07$ & 1017 & 329 & $12 \cdot 2 \%$ & $10 \cdot 0-14 \cdot 9$ & 1008 \\
\hline Adenocarcinoma with variants of trachea & 0.01 & $0.01-0.01$ & 164 & 53 & $15 \cdot 7 \%$ & $10 \cdot 3-24 \cdot 0$ & 158 \\
\hline Salivary-gland-type tumours of trachea & 0.01 & $0.01-0.01$ & 175 & 57 & $70 \cdot 1 \%$ & $62 \cdot 0-79 \cdot 2$ & 174 \\
\hline Rare epithelial tumour of lung & $4 \cdot 37$ & $4 \cdot 34-4 \cdot 40$ & 68452 & 24930 & $14 \cdot 9 \%$ & $14 \cdot 6-15 \cdot 2$ & 67936 \\
\hline Adenosquamous carcinoma of lung & 0.29 & $0 \cdot 29-0 \cdot 30$ & 4607 & 1492 & $21 \cdot 9 \%$ & $20 \cdot 5-23 \cdot 4$ & 4566 \\
\hline Large-cell carcinoma of lung & 3.81 & $3 \cdot 78-3 \cdot 84$ & 59714 & 19342 & $13 \cdot 9 \%$ & $13 \cdot 5-14 \cdot 2$ & 59332 \\
\hline Salivary-gland-type tumours of lung & 0.06 & $0.05-0.06$ & 879 & 285 & $40 \cdot 4 \%$ & $36 \cdot 8-44 \cdot 4$ & 866 \\
\hline \multirow[t]{2}{*}{ Sarcomatoid carcinoma of lung } & 0.21 & $0.20-0.22$ & 3255 & 1054 & $17 \cdot 5 \%$ & $16 \cdot 0-19 \cdot 2$ & 3183 \\
\hline & & & & & & \multicolumn{2}{|c|}{ (Table 1 continues on next page) } \\
\hline
\end{tabular}




\begin{tabular}{|c|c|c|c|c|c|c|c|}
\hline & $\begin{array}{l}\text { Crude incidence } \\
\text { rate per } \\
100000 \text { people } \\
\text { per year }\end{array}$ & $95 \% \mathrm{Cl}$ & $\begin{array}{l}\text { Observed cases } \\
\text { in } 83 \text { cancer } \\
\text { registries in } \\
2000-07\end{array}$ & $\begin{array}{l}\text { Estimated } \\
\text { new cases in } \\
2013 \text { in EU28 }\end{array}$ & $\begin{array}{l}5 \text {-year } \\
\text { relative } \\
\text { survival (\%) }\end{array}$ & $95 \% \mathrm{Cl}$ & $\begin{array}{l}\text { Observed cases } \\
\text { in } 94 \text { cancer } \\
\text { registries in } \\
2000-07\end{array}$ \\
\hline \multicolumn{8}{|l|}{ (Continued from previous page) } \\
\hline Epithelial tumours of thymus & 0.18 & $0 \cdot 17-0 \cdot 19$ & 2795 & 905 & $64 \cdot 3 \%$ & $62 \cdot 1-66 \cdot 6$ & 2729 \\
\hline Malignant thymoma & 0.14 & $0.14-0.15$ & 2268 & 735 & $69 \cdot 3 \%$ & $67 \cdot 0-71 \cdot 8$ & 2248 \\
\hline Squamous cell carcinoma of thymus & 0.01 & $0.01-0.01$ & 114 & 37 & $40 \cdot 4 \%$ & $30 \cdot 4-53 \cdot 7$ & 112 \\
\hline Undifferentiated carcinoma of thymus & 0.00 & $0.00-0.00$ & 36 & 12 & $13 \cdot 3 \%$ & $5 \cdot 1-34 \cdot 8$ & 36 \\
\hline Lymphoepithelial carcinoma of thymus & 0.00 & $0.00-0.00$ & 12 & 4 & $55 \cdot 0 \%$ & $29 \cdot 2-103 \cdot 6$ & 11 \\
\hline Adenocarcinoma with variants of thymus & 0.00 & $0.00-0 \cdot 00$ & 45 & 15 & $37 \cdot 3 \%$ & $21 \cdot 7-64 \cdot 1$ & 44 \\
\hline Malignant mesothelioma & $2 \cdot 14$ & $2 \cdot 12-2 \cdot 16$ & 33552 & 10868 & $5 \cdot 3 \%$ & $4 \cdot 9-5 \cdot 6$ & 32330 \\
\hline Mesothelioma of pleura and pericardium & 1.83 & $1.81-1.85$ & 28676 & 9288 & $4 \cdot 6 \%$ & $4 \cdot 2-4 \cdot 9$ & 27893 \\
\hline Mesothelioma of peritoneum and tunica vaginalis & 0.13 & $0.13-0.14$ & 2065 & 669 & $13 \cdot 2 \%$ & 11.5-15.1 & 1965 \\
\hline Rare: female genital cancers & 22.73 & $22 \cdot 66-22 \cdot 81$ & 356151 & 113796 & $57 \cdot 7 \%$ & $57 \cdot 5-57 \cdot 9$ & 347015 \\
\hline Rare epithelial tumours of breast & $4 \cdot 12$ & $4 \cdot 09-4 \cdot 16$ & 64605 & 22980 & $91 \cdot 4 \%$ & $91 \cdot 0-91 \cdot 8$ & 64368 \\
\hline Mammary Paget's disease of breast & 0.41 & $0 \cdot 40-0 \cdot 42$ & 6488 & 2101 & $85.9 \%$ & $84 \cdot 6-87 \cdot 3$ & 6508 \\
\hline Special types of adenocarcinoma of breast & 3.06 & $3.04-3.09$ & 48012 & 15551 & $95 \cdot 2 \%$ & $94 \cdot 8-95 \cdot 6$ & 47974 \\
\hline Metaplastic carcinoma of breast & 0.10 & $0 \cdot 10-0 \cdot 11$ & 1576 & 510 & $65.0 \%$ & $61 \cdot 9-68 \cdot 3$ & 1583 \\
\hline Salivary-gland-type tumours of breast & 0.06 & $0.05-0.06$ & 868 & 281 & $90 \cdot 9 \%$ & $87 \cdot 6-94 \cdot 2$ & 870 \\
\hline Epithelial tumour of male breast & 0.52 & $0.51-0.53$ & 8098 & 5376 & $77 \cdot 0 \%$ & $75 \cdot 5-78 \cdot 5$ & 7882 \\
\hline Rare epithelial tumours of corpus uteri & 0.70 & $0.69-0.72$ & 11038 & 3932 & $44: 3 \%$ & $43 \cdot 2-45 \cdot 5$ & 11013 \\
\hline Squamous cell carcinoma with variants of corpus uteri & 0.06 & $0.06-0.07$ & 1003 & 325 & $58 \cdot 2 \%$ & $54 \cdot 6-62 \cdot 1$ & 989 \\
\hline Adenoid cystic carcinoma of corpus uteri & 0.00 & $0.00-0.00$ & 5 & 2 & $64 \cdot 1 \%$ & $31 \cdot 3-131 \cdot 1$ & 5 \\
\hline $\begin{array}{l}\text { Clear cell adenocarcinoma, not otherwise specified of corpus } \\
\text { uteri }\end{array}$ & 0.16 & $0 \cdot 16-0 \cdot 17$ & 2527 & 819 & $58 \cdot 6 \%$ & $56 \cdot 2-61 \cdot 2$ & 2532 \\
\hline Serous (papillary) carcinoma of corpus uteri & 0.08 & $0.07-0.08$ & 1227 & 397 & $40 \cdot 0 \%$ & $36 \cdot 5-43 \cdot 9$ & 1225 \\
\hline Mullerian mixed tumour of corpus uteri & 0.40 & $0.39-0.41$ & 6276 & 2033 & $36.9 \%$ & $35 \cdot 5-38 \cdot 4$ & 6263 \\
\hline Epithelial tumours of cervix uteri & 6.28 & $6 \cdot 24-6 \cdot 32$ & 98321 & 28898 & $65 \cdot 4 \%$ & $65 \cdot 1-65 \cdot 8$ & 96821 \\
\hline Squamous cell carcinoma with variants of cervix uteri & 4.73 & $4 \cdot 70-4 \cdot 76$ & 74105 & 24003 & $66.8 \%$ & $66 \cdot 5-67 \cdot 2$ & 73810 \\
\hline Adenocarcinoma with variants of cervix uteri & 0.91 & $0.89-0.92$ & 14252 & 4616 & $67 \cdot 4 \%$ & $66 \cdot 5-68 \cdot 3$ & 14221 \\
\hline Undifferentiated carcinoma of cervix uteri & 0.03 & $0.03-0.03$ & 480 & 155 & $35 \cdot 3 \%$ & $30 \cdot 9-40 \cdot 4$ & 478 \\
\hline Mullerian mixed tumour of cervix uteri & 0.02 & $0.01-0.02$ & 257 & 83 & $34 \cdot 3 \%$ & $28 \cdot 1-41 \cdot 7$ & 256 \\
\hline Epithelial tumour of ovary and fallopian tube & $9 \cdot 38$ & $9 \cdot 33-9 \cdot 43$ & 146908 & 45382 & $37 \cdot 5 \%$ & $37 \cdot 2-37 \cdot 8$ & 141240 \\
\hline Adenocarcinoma with variants of ovary & 5.95 & $5.92-5.99$ & 93263 & 30208 & $38.7 \%$ & $38 \cdot 3-39 \cdot 1$ & 92814 \\
\hline Mucinous adenocarcinoma of ovary & 0.77 & $0.76-0.78$ & 12066 & 3908 & $59 \cdot 9 \%$ & $58 \cdot 9-60 \cdot 9$ & 12010 \\
\hline Clear cell adenocarcinoma of ovary & 0.30 & $0 \cdot 29-0 \cdot 31$ & 4753 & 1540 & $55 \cdot 5 \%$ & $53 \cdot 8-57 \cdot 2$ & 4761 \\
\hline Primary peritoneal serous or papillary carcinoma of ovary & 0.08 & $0.08-0.09$ & 1280 & 415 & $21 \cdot 9 \%$ & $19 \cdot 1-25 \cdot 2$ & 1280 \\
\hline Mullerian mixed tumour of ovary & 0.14 & $0 \cdot 14-0.15$ & 2255 & 730 & $21 \cdot 4 \%$ & $19 \cdot 5-23 \cdot 6$ & 2242 \\
\hline Adenocarcinoma with variant of fallopian tube & 0.17 & $0 \cdot 16-0 \cdot 18$ & 2683 & 869 & $59 \cdot 1 \%$ & $56 \cdot 8-61 \cdot 6$ & 2672 \\
\hline Non-epithelial tumours of ovary & 0.25 & $0.25-0.26$ & 3977 & 1288 & $82 \cdot 0 \%$ & $80 \cdot 6-83 \cdot 5$ & 3970 \\
\hline Sex cord tumours of ovary & 0.13 & $0.12-0.13$ & 2006 & 650 & $78.8 \%$ & $76 \cdot 5-81 \cdot 1$ & 1998 \\
\hline Malignant or immature teratomas of ovary & 0.05 & $0.05-0.06$ & 833 & 270 & $83 \cdot 4 \%$ & $80 \cdot 6-86 \cdot 3$ & 829 \\
\hline Germ cell tumour of ovary & 0.07 & $0.07-0.08$ & 1138 & 369 & $86.6 \%$ & $84 \cdot 4-88 \cdot 8$ & 1143 \\
\hline Epithelial tumours of vulva and vagina & 1.97 & $1.95-2 \cdot 00$ & 30938 & 11215 & $58 \cdot 1 \%$ & $57 \cdot 3-58 \cdot 8$ & 30238 \\
\hline Squamous cell carcinoma with variants of vulva and vagina & 1.69 & $1 \cdot 67-1 \cdot 71$ & 26422 & 8558 & $59.8 \%$ & $59 \cdot 0-60 \cdot 7$ & 26277 \\
\hline Adenocarcinoma with variants of vulva and vagina & 0.07 & $0.07-0.08$ & 1120 & 363 & $45 \cdot 8 \%$ & $42 \cdot 3-49 \cdot 6$ & 1112 \\
\hline Paget's disease of vulva and vagina & 0.05 & $0.04-0.05$ & 746 & 242 & $88.0 \%$ & $83 \cdot 7-92 \cdot 6$ & 744 \\
\hline Undifferentiated carcinoma of vulva and vagina & 0.01 & $0.00-0.01$ & 85 & 28 & $25 \cdot 6 \%$ & $15 \cdot 8-41 \cdot 6$ & 85 \\
\hline Trophoblastic tumour of placenta & 0.02 & $0.02-0.03$ & 363 & 100 & $89 \cdot 3 \%$ & $85 \cdot 3-92 \cdot 2$ & 361 \\
\hline Choriocarcinoma of placenta & 0.02 & $0.02-0.02$ & 352 & 114 & $89.8 \%$ & $86 \cdot 5-93 \cdot 3$ & 350 \\
\hline
\end{tabular}




\begin{tabular}{|c|c|c|c|c|c|c|c|}
\hline & $\begin{array}{l}\text { Crude incidence } \\
\text { rate per } \\
100000 \text { people } \\
\text { per year }\end{array}$ & $95 \% \mathrm{Cl}$ & $\begin{array}{l}\text { Observed cases } \\
\text { in } 83 \text { cancer } \\
\text { registries in } \\
2000-07\end{array}$ & $\begin{array}{l}\text { Estimated } \\
\text { new cases in } \\
2013 \text { in EU28 }\end{array}$ & $\begin{array}{l}\text { 5-year } \\
\text { relative } \\
\text { survival (\%) }\end{array}$ & $95 \% \mathrm{Cl}$ & $\begin{array}{l}\text { Observed cases } \\
\text { in } 94 \text { cancer } \\
\text { registries in } \\
2000-07\end{array}$ \\
\hline \multicolumn{8}{|l|}{ (Continued from previous page) } \\
\hline Rare: male genital and urogenital cancers & 7.09 & $7 \cdot 05-7 \cdot 14$ & 111128 & 38138 & $73.6 \%$ & $73 \cdot 3-74 \cdot 0$ & 109102 \\
\hline Rare epithelial tumours of prostate & 0.60 & $0.59-0.61$ & 9437 & 3563 & $75 \cdot 4 \%$ & $74 \cdot 0-76 \cdot 9$ & 9291 \\
\hline Squamous cell carcinoma with variants of prostate & 0.02 & $0.02-0.02$ & 291 & 94 & $41 \cdot 1 \%$ & $34 \cdot 1-49 \cdot 5$ & 287 \\
\hline Infiltrating duct carcinoma of prostate & 0.51 & $0.50-0.53$ & 8064 & 2612 & $78 \cdot 7 \%$ & $77 \cdot 2-80 \cdot 3$ & 7945 \\
\hline Transitional cell carcinoma of prostate & 0.06 & $0.06-0.07$ & 960 & 311 & $57 \cdot 7 \%$ & $53 \cdot 4-62 \cdot 4$ & 941 \\
\hline Salivary-gland-type tumours of prostate & 0.01 & $0.01-0.01$ & 122 & 40 & $78 \cdot 5 \%$ & $64 \cdot 4-95 \cdot 7$ & 118 \\
\hline Testicular and paratesticular cancers & $3 \cdot 29$ & $3 \cdot 27-3 \cdot 32$ & 51605 & 16061 & $94.9 \%$ & $94 \cdot 7-95 \cdot 2$ & 51011 \\
\hline Paratesticular adenocarcinoma with variants & 0.00 & $0.00-0.00$ & 22 & 7 & $82 \cdot 5 \%$ & $65 \cdot 3-104 \cdot 1$ & 22 \\
\hline Non-seminomatous testicular cancer & $1 \cdot 27$ & $1 \cdot 25-1 \cdot 28$ & 19835 & 6425 & $92 \cdot 9 \%$ & $92 \cdot 5-93 \cdot 3$ & 19714 \\
\hline Seminomatous testicular cancer & 1.82 & $1 \cdot 80-1 \cdot 84$ & 28516 & 9236 & $97 \cdot 5 \%$ & $97 \cdot 3-97 \cdot 8$ & 28326 \\
\hline Spermatocytic seminoma & 0.03 & $0.03-0.03$ & 502 & 163 & $95 \cdot 3 \%$ & $91 \cdot 8-99 \cdot 0$ & 502 \\
\hline Teratoma with malignant transformation & 0.00 & $0.00-0.00$ & 20 & 6 & $91 \cdot 4 \%$ & $78 \cdot 6-106 \cdot 2$ & 20 \\
\hline Testicular sex cord cancer & 0.02 & $0.02-0.02$ & 340 & 110 & $82 \cdot 3 \%$ & $77 \cdot 3-87 \cdot 6$ & 337 \\
\hline Epithelial tumours of penis & 0.66 & $0.65-0.67$ & 10368 & 3887 & $67 \cdot 5 \%$ & $66 \cdot 2-68 \cdot 9$ & 10210 \\
\hline Squamous cell carcinoma with variants of penis & 0.62 & $0.60-0.63$ & 9646 & 3124 & $68.9 \%$ & $67 \cdot 5-70 \cdot 2$ & 9621 \\
\hline Adenocarcinoma with variants of penis & 0.01 & $0.00-0.01$ & 88 & 29 & $49 \cdot 0 \%$ & $36 \cdot 2-66 \cdot 4$ & 86 \\
\hline Rare epithelial tumours of kidney & 0.05 & $0.04-0.05$ & 723 & 261 & $18 \cdot 8 \%$ & $15 \cdot 8-22 \cdot 4$ & 704 \\
\hline Squamous cell carcinoma spindle cell type of kidney & 0.01 & $0.01-0.01$ & 190 & 62 & $22 \cdot 0 \%$ & $16 \cdot 0-30 \cdot 2$ & 190 \\
\hline Squamous cell carcinoma with variants of kidney & 0.03 & $0.03-0.04$ & 533 & 173 & $17.7 \%$ & $14 \cdot 4-21 \cdot 7$ & 514 \\
\hline Epithelial tumours of pelvis and ureter & 1.58 & $1.57-1 \cdot 60$ & 24826 & 9187 & $48 \cdot 8 \%$ & $48 \cdot 0-49 \cdot 7$ & 24017 \\
\hline Transitional cell carcinoma of pelvis and ureter & 1.41 & $1 \cdot 39-1 \cdot 43$ & 22099 & 7158 & $51 \cdot 3 \%$ & $50 \cdot 4-52 \cdot 2$ & 21607 \\
\hline Squamous cell carcinoma with variants of pelvis and ureter & 0.02 & $0.02-0.03$ & 372 & 121 & $15 \cdot 0 \%$ & $11 \cdot 2-20 \cdot 2$ & 366 \\
\hline Adenocarcinoma with variants of pelvis and ureter & 0.02 & $0.02-0.02$ & 326 & 106 & $43.0 \%$ & $36 \cdot 7-50 \cdot 5$ & 320 \\
\hline Epithelial tumours of urethra & 0.13 & $0 \cdot 13-0.14$ & 2077 & 784 & $44.5 \%$ & $41 \cdot 6-47 \cdot 5$ & 2050 \\
\hline Transitional cell carcinoma of urethra & 0.09 & $0.08-0.09$ & 1390 & 450 & $42 \cdot 9 \%$ & $39 \cdot 5-46 \cdot 7$ & 1387 \\
\hline Squamous cell carcinoma with variants of urethra & 0.02 & $0.02-0.02$ & 329 & 107 & $51 \cdot 1 \%$ & $44 \cdot 6-58 \cdot 5$ & 329 \\
\hline Adenocarcinoma with variants of urethra & 0.01 & $0.01-0.01$ & 190 & 62 & $52 \cdot 0 \%$ & $43 \cdot 2-62 \cdot 6$ & 189 \\
\hline Rare epithelial tumours of bladder & 0.65 & $0.64-0.67$ & 10226 & 3819 & $32 \cdot 3 \%$ & $31 \cdot 2-33 \cdot 5$ & 10152 \\
\hline Squamous cell carcinoma with variants of bladder & 0.36 & $0.35-0.36$ & 5566 & 1803 & $24 \cdot 3$ & $22 \cdot 9-25 \cdot 7$ & 5534 \\
\hline Adenocarcinoma with variants of bladder & $0 \cdot 30$ & $0 \cdot 29-0.31$ & 4653 & 1507 & $41 \cdot 9 \%$ & $40 \cdot 1-43 \cdot 8$ & 4614 \\
\hline Salivary-gland-type tumours of bladder & 0.00 & $0.00-0.00$ & 7 & 2 & NE & NE & 7 \\
\hline Extragonadal germ cell tumours & 0.12 & $0.11-0.12$ & 1862 & 576 & $69 \cdot 6 \%$ & $67 \cdot 3-71 \cdot 8$ & 1851 \\
\hline Non-seminomatous germ cell tumours & 0.06 & $0.05-0.06$ & 915 & 296 & $62 \cdot 5 \%$ & $59 \cdot 2-66 \cdot 0$ & 909 \\
\hline Seminomatous germ cell tumours & 0.01 & $0.01-0.01$ & 130 & 42 & $85.9 \%$ & $79 \cdot 1-93 \cdot 3$ & 130 \\
\hline Germ cell tumours of CNS & 0.04 & $0.03-0.04$ & 574 & 186 & $82.5 \%$ & $79 \cdot 2-85 \cdot 9$ & 572 \\
\hline Rare: neuroendocrine tumours & 3.51 & $3 \cdot 43-3 \cdot 58$ & 54942 & 19587 & $53 \cdot 5 \%$ & $53 \cdot 0-54 \cdot 1$ & 54331 \\
\hline $\begin{array}{l}\text { GEP, well differentiated not functioning endocrine carcinoma of } \\
\text { pancreas and digestive tract }\end{array}$ & 1.01 & $1.00-1.03$ & 15852 & 5134 & $72 \cdot 0 \%$ & $71 \cdot 1-73 \cdot 0$ & 15656 \\
\hline $\begin{array}{l}\text { GEP, well differentiated functioning endocrine carcinoma of } \\
\text { pancreas and digestive tract }\end{array}$ & 0.03 & $0.02-0.03$ & 411 & 133 & $61 \cdot 3 \%$ & $55 \cdot 9-67 \cdot 3$ & 407 \\
\hline GEP, poorly differentiated endocrine carcinoma & 0.67 & $0.65-0.68$ & 10421 & 3375 & $35 \cdot 0 \%$ & $33 \cdot 9-36 \cdot 2$ & 10456 \\
\hline GEP, mixed endocrine-exocrine carcinoma & 0.01 & $0.01-0.01$ & 147 & 48 & $25 \cdot 9 \%$ & $18 \cdot 2-37 \cdot 0$ & 141 \\
\hline Endocrine carcinoma of thyroid gland & 0.24 & $0.23-0.25$ & 3796 & 1230 & $83.6 \%$ & $82 \cdot 1-85 \cdot 2$ & 3793 \\
\hline Neuroendocrine carcinoma of skin & 0.19 & $0 \cdot 19-0 \cdot 20$ & 3024 & 979 & $55 \cdot 9 \%$ & $53 \cdot 2-58 \cdot 7$ & 2997 \\
\hline Typical and atypical carcinoid of the lung & 0.39 & $0 \cdot 38-0 \cdot 40$ & 6160 & 1995 & $81 \cdot 1 \%$ & $79 \cdot 9-82 \cdot 5$ & 6058 \\
\hline Neuroendocrine carcinoma of other sites & 0.90 & $0.89-0.92$ & 14120 & 4573 & $23.9 \%$ & $23 \cdot 0-24 \cdot 8$ & 13958 \\
\hline Pheochromocytoma, malignant & 0.04 & $0.04-0.04$ & 650 & 211 & $70 \cdot 1 \%$ & $65 \cdot 9-74 \cdot 5$ & 612 \\
\hline \multirow[t]{2}{*}{ Paraganglioma } & 0.02 & $0.02-0.02$ & 347 & 112 & $56 \cdot 3 \%$ & $50 \cdot 6-62 \cdot 6$ & 342 \\
\hline & & & & & & \multicolumn{2}{|c|}{ (Table 1 continues on next page) } \\
\hline
\end{tabular}




\begin{tabular}{|c|c|c|c|c|c|c|c|}
\hline & $\begin{array}{l}\text { Crude incidence } \\
\text { rate per } \\
100000 \text { people } \\
\text { per year }\end{array}$ & $95 \% \mathrm{Cl}$ & $\begin{array}{l}\text { Observed cases } \\
\text { in } 83 \text { cancer } \\
\text { registries in } \\
2000-07\end{array}$ & $\begin{array}{l}\text { Estimated } \\
\text { new cases in } \\
2013 \text { in EU28 }\end{array}$ & $\begin{array}{l}\text { 5-year } \\
\text { relative } \\
\text { survival (\%) }\end{array}$ & $95 \% \mathrm{Cl}$ & $\begin{array}{l}\text { Observed cases } \\
\text { in } 94 \text { cancer } \\
\text { registries in } \\
2000-07\end{array}$ \\
\hline \multicolumn{8}{|l|}{ (Continued from previous page) } \\
\hline Rare: cancers of the endocrine organs & $5 \cdot 35$ & $5 \cdot 32-5 \cdot 39$ & 83836 & 28322 & $88.1 \%$ & $87.8-88 \cdot 4$ & 82523 \\
\hline Carcinomas of pituitary gland & 0.04 & $0.03-0.04$ & 582 & 206 & $63 \cdot 7 \%$ & $58 \cdot 9-69 \cdot 0$ & 511 \\
\hline Carcinomas of thyroid gland & 5.07 & $5 \cdot 03-5 \cdot 10$ & 79418 & 26768 & $90 \cdot 5 \%$ & $90 \cdot 2-90 \cdot 8$ & 78533 \\
\hline Carcinomas of parathyroid gland & 0.03 & $0.02-0.03$ & 410 & 143 & $80.8 \%$ & $75 \cdot 8-86 \cdot 2$ & 395 \\
\hline Carcinomas of adrenal gland & 0.22 & $0.21-0.23$ & 3424 & 1205 & $32 \cdot 1 \%$ & $30 \cdot 2-34 \cdot 0$ & 3103 \\
\hline Rare: sarcomas & 5.86 & $5 \cdot 83-6 \cdot 00$ & 91878 & 31916 & $59 \cdot 5 \%$ & $57 \cdot 4-58 \cdot 2$ & 90568 \\
\hline Soft tissue sarcoma & 4.71 & $4 \cdot 68-4 \cdot 74$ & 73795 & 25851 & $56 \cdot 7 \%$ & $56 \cdot 3-57 \cdot 1$ & 72696 \\
\hline Soft tissue sarcoma of head and neck & 0.26 & $0.25-0.27$ & 4087 & 1324 & $59 \cdot 8 \%$ & $57 \cdot 7-61 \cdot 8$ & 4062 \\
\hline Soft tissue sarcoma of limbs & $1 \cdot 10$ & $1 \cdot 08-1 \cdot 11$ & 17178 & 5564 & $67 \cdot 7 \%$ & $66 \cdot 8-68 \cdot 6$ & 17094 \\
\hline Soft tissue sarcoma of superficial trunk & 0.50 & $0.49-0.51$ & 7813 & 2531 & $48 \cdot 1 \%$ & $46 \cdot 8-49 \cdot 5$ & 7723 \\
\hline Soft tissue sarcoma of mediastinum & 0.03 & $0.03-0.03$ & 465 & 151 & $23 \cdot 4 \%$ & $19 \cdot 3-28 \cdot 3$ & 457 \\
\hline Soft tissue sarcoma of heart & 0.01 & $0.01-0.02$ & 216 & 70 & $14 \cdot 4 \%$ & $9 \cdot 8-21 \cdot 0$ & 203 \\
\hline Soft tissue sarcoma of breast & 0.18 & $0 \cdot 18-0 \cdot 19$ & 2865 & 928 & $74 \cdot 5 \%$ & $72 \cdot 5-76 \cdot 5$ & 2864 \\
\hline Soft tissue sarcoma of uterus & 0.55 & $0.54-0.56$ & 8657 & 2804 & $52 \cdot 0 \%$ & $50 \cdot 8-53 \cdot 2$ & 8568 \\
\hline Other soft tissue sarcomas of genitourinary tract & 0.20 & $0 \cdot 19-0 \cdot 21$ & 3160 & 1024 & $50 \cdot 4 \%$ & $48 \cdot 3-52 \cdot 5$ & 3107 \\
\hline Soft tissue sarcoma of viscera & 0.38 & $0 \cdot 37-0 \cdot 39$ & 6004 & 1945 & $42 \cdot 1 \%$ & $40 \cdot 6-43 \cdot 6$ & 5915 \\
\hline Soft tissue sarcoma of paratestis & 0.03 & $0.03-0.04$ & 510 & 165 & $87 \cdot 2 \%$ & $82 \cdot 2-92 \cdot 4$ & 510 \\
\hline Soft tissue sarcoma of retroperitoneum and peritoneum & 0.31 & $0 \cdot 30-0 \cdot 32$ & 4911 & 1591 & $38.8 \%$ & $37 \cdot 1-40 \cdot 5$ & 4854 \\
\hline Soft tissue sarcoma of pelvis & 0.20 & $0.19-0.20$ & 3090 & 1001 & $47 \cdot 4 \%$ & $45 \cdot 3-49 \cdot 6$ & 3064 \\
\hline Soft tissue sarcoma of skin & 0.30 & $0.29-0.31$ & 4737 & 1534 & $90 \cdot 2 \%$ & $88 \cdot 8-91 \cdot 7$ & 4728 \\
\hline Soft tissue sarcoma of paraorbit & 0.01 & $0.01-0.01$ & 117 & 38 & $63.3 \%$ & $52 \cdot 9-75 \cdot 7$ & 115 \\
\hline Soft tissue sarcoma of brain and other parts of nervous system & 0.17 & $0 \cdot 17-0.18$ & 2723 & 882 & $54 \cdot 5 \%$ & $52 \cdot 3-56 \cdot 7$ & 2695 \\
\hline Embryonal rhabdomyosarcoma of soft tissue & 0.05 & $0.05-0.06$ & 836 & 271 & $66 \cdot 2 \%$ & $62 \cdot 8-69 \cdot 8$ & 825 \\
\hline Alveolar rhabdomyosarcoma of soft tissue & 0.03 & $0.03-0.04$ & 519 & 168 & $36.0 \%$ & $31 \cdot 7-40 \cdot 8$ & 515 \\
\hline Ewing's sarcoma of soft tissue & 0.06 & $0.06-0.07$ & 998 & 323 & $44 \cdot 9 \%$ & $41 \cdot 5-48 \cdot 5$ & 992 \\
\hline Bone sarcoma & 0.85 & $0.84-0.87$ & 13376 & 4382 & $58.6 \%$ & $57 \cdot 6-59 \cdot 6$ & 13216 \\
\hline Osteogenic sarcoma & 0.21 & $0.21-0.22$ & 3330 & 1079 & $51 \cdot 4 \%$ & $49 \cdot 5-53 \cdot 4$ & 3282 \\
\hline Chondrogenic sarcomas & 0.26 & $0.25-0.27$ & 4107 & 1330 & $70 \cdot 0 \%$ & $68 \cdot 2-71 \cdot 7$ & 4060 \\
\hline Notochordal sarcomas, chordoma & 0.07 & $0.07-0.08$ & 1145 & 371 & $62 \cdot 5 \%$ & $58 \cdot 2-67 \cdot 2$ & 755 \\
\hline Vascular sarcomas & 0.01 & $0.01-0.01$ & 129 & 42 & $45 \cdot 1 \%$ & $36 \cdot 4-55 \cdot 9$ & 129 \\
\hline Ewing's sarcoma & 0.12 & $0.12-0.13$ & 1943 & 629 & $52 \cdot 8 \%$ & $50 \cdot 4-55 \cdot 3$ & 1932 \\
\hline Epithelial tumours, adamantinoma & 0.01 & $0.01-0.02$ & 213 & 69 & $87 \cdot 2 \%$ & $81 \cdot 0-93 \cdot 9$ & 210 \\
\hline $\begin{array}{l}\text { Other high grade sarcomas (fibrosarcoma, malignant fibrous } \\
\text { histiocytoma) }\end{array}$ & 0.02 & $0.02-0.02$ & 304 & 98 & $46 \cdot 2 \%$ & $40 \cdot 1-53 \cdot 1$ & 302 \\
\hline Gastrointestinal stromal sarcoma & 0.30 & $0 \cdot 29-0 \cdot 31$ & 4706 & 1683 & $72 \cdot 3 \%$ & $70 \cdot 4-74 \cdot 1$ & 4781 \\
\hline Rare: cancers of the CNS & $7 \cdot 56$ & $7 \cdot 51-8.00$ & 118391 & 36343 & $21 \cdot 3 \%$ & $21 \cdot 0-21 \cdot 6$ & 111838 \\
\hline Tumours of CNS & $7 \cdot 36$ & $7 \cdot 32-7 \cdot 40$ & 115289 & 35339 & $20 \cdot 3 \%$ & $20 \cdot 0-20 \cdot 6$ & 108752 \\
\hline Astrocytic tumours of CNS & 4.99 & $4 \cdot 95-5 \cdot 02$ & 78118 & 25303 & $15 \cdot 0 \%$ & $14 \cdot 8-15 \cdot 3$ & 77195 \\
\hline Oligodendroglial tumours of CNS & 0.39 & $0 \cdot 38-0 \cdot 40$ & 6148 & 1991 & $51 \cdot 8 \%$ & $50 \cdot 4-53 \cdot 3$ & 6124 \\
\hline Ependymal tumours of CNS & 0.21 & $0.20-0.21$ & 3212 & 1040 & $72 \cdot 7 \%$ & $71 \cdot 0-74 \cdot 5$ & 3190 \\
\hline Choroid plexus carcinoma of CNS & 0.01 & $0.01-0.01$ & 98 & 32 & $57 \cdot 7 \%$ & $48 \cdot 3-68 \cdot 8$ & 95 \\
\hline Malignant meningiomas & 0.16 & $0 \cdot 16-0 \cdot 17$ & 2564 & 830 & $61 \cdot 1 \%$ & $58 \cdot 8-63 \cdot 4$ & 2509 \\
\hline Embryonal tumours of CNS & 0.20 & $0 \cdot 19-0 \cdot 21$ & 3102 & 1005 & $56 \cdot 1 \%$ & $54 \cdot 2-58 \cdot 1$ & 3092 \\
\hline Rare: skin cancers and non-cutaneous melanoma & $1 \cdot 22$ & $1 \cdot 18-1 \cdot 25$ & 21878 & 7086 & $70 \cdot 2 \%$ & $69 \cdot 3-71 \cdot 1$ & 21637 \\
\hline Malignant melanoma of mucosa & 0.15 & $0 \cdot 14-0 \cdot 15$ & 2279 & 738 & $20 \cdot 3 \%$ & $18 \cdot 3-22 \cdot 6$ & 2277 \\
\hline Malignant melanoma of uvea & 0.70 & $0.69-0.72$ & 11022 & 3570 & $71.0 \%$ & $69 \cdot 8-72 \cdot 2$ & 10872 \\
\hline Adnexal carcinoma of skin & 0.30 & $0.29-0.31$ & 4684 & 1517 & $86.1 \%$ & $83 \cdot 9-88 \cdot 0$ & 4661 \\
\hline Kaposi's sarcoma & 0.25 & $0.24-0.26$ & 3893 & 1261 & $78.9 \%$ & $77 \cdot 1-80 \cdot 8$ & 3830 \\
\hline
\end{tabular}




\begin{tabular}{|c|c|c|c|c|c|c|c|}
\hline & $\begin{array}{l}\text { Crude incidence } \\
\text { rate per } \\
100000 \text { people } \\
\text { per year }\end{array}$ & $95 \% \mathrm{Cl}$ & $\begin{array}{l}\text { Observed cases } \\
\text { in } 83 \text { cancer } \\
\text { registries in } \\
2000-07\end{array}$ & $\begin{array}{l}\text { Estimated } \\
\text { new cases in } \\
2013 \text { in EU28 }\end{array}$ & $\begin{array}{l}5 \text {-year } \\
\text { relative } \\
\text { survival (\%) }\end{array}$ & $95 \% \mathrm{Cl}$ & $\begin{array}{l}\text { Observed cases } \\
\text { in } 94 \text { cancer } \\
\text { registries in } \\
2000-07\end{array}$ \\
\hline \multicolumn{8}{|l|}{ (Continued from previous page) } \\
\hline Rare: embryonal tumours & 0.34 & $0 \cdot 33-0 \cdot 35$ & 5363 & 1822 & $78.6 \%$ & $77 \cdot 4-79 \cdot 8$ & 5239 \\
\hline Neuroblastoma and ganglioneuroblastoma & 0.10 & $0 \cdot 10-0.11$ & 1566 & 507 & $64 \cdot 6 \%$ & $62 \cdot 1-67 \cdot 3$ & 1553 \\
\hline Nephroblastoma & 0.13 & $0 \cdot 12-0 \cdot 13$ & 1965 & 636 & $88 \cdot 2 \%$ & $86 \cdot 6-89 \cdot 7$ & 1936 \\
\hline Retinoblastoma & 0.05 & $0.05-0.06$ & 860 & 279 & $96 \cdot 5 \%$ & $95 \cdot 1-97 \cdot 9$ & 801 \\
\hline Hepatoblastoma & 0.02 & $0.02-0.03$ & 357 & 116 & $76 \cdot 8 \%$ & $72 \cdot 2-81 \cdot 7$ & 352 \\
\hline Pleuropulmonary blastoma & 0.00 & $0.00-0.00$ & 9 & 3 & $53 \cdot 5 \%$ & $28 \cdot 3-101 \cdot 1$ & 9 \\
\hline Pancreatoblastoma & 0.00 & $0.00-0.00$ & 39 & 13 & $34 \cdot 3 \%$ & $20 \cdot 7-56 \cdot 9$ & 35 \\
\hline Olfactory neuroblastoma & 0.03 & $0.03-0.03$ & 498 & 161 & $64.0 \%$ & $59 \cdot 2-69 \cdot 2$ & 489 \\
\hline Odontogenic malignant tumours & 0.00 & $0.00-0.01$ & 69 & 22 & $61 \cdot 6 \%$ & $49 \cdot 0-77 \cdot 5$ & 69 \\
\hline Rare: haematological malignancies & 27.73 & $27 \cdot 65-27 \cdot 82$ & 434469 & 156099 & $50 \cdot 5 \%$ & $50 \cdot 3-50 \cdot 7$ & 423741 \\
\hline Rare lymphoid diseases & 18.09 & $18 \cdot 02-18 \cdot 16$ & 283399 & 100343 & $55 \cdot 8 \%$ & $55 \cdot 5-56 \cdot 0$ & 279794 \\
\hline Hodgkin's lymphoma, classical & 2.46 & $2 \cdot 44-2 \cdot 49$ & 38588 & 12499 & $81 \cdot 4 \%$ & $80 \cdot 9-81 \cdot 8$ & 38389 \\
\hline Hodgkin's lymphoma nodular lymphocyte predominance & 0.09 & $0.09-0 \cdot 10$ & 1483 & 480 & $93.6 \%$ & $91 \cdot 8-95 \cdot 3$ & 1507 \\
\hline $\begin{array}{l}\text { Precursor B-cell or T-cell lymphoblastic leukaemia or } \\
\text { lymphoma (and Burkitt's leukaemia or lymphoma) }\end{array}$ & 1.46 & $1 \cdot 44-1 \cdot 47$ & 22795 & 7383 & $58 \cdot 1 \%$ & $57 \cdot 4-58 \cdot 8$ & 22496 \\
\hline $\begin{array}{l}\text { T-cell cutaneous lymphoma (Sezary syndrome, mycosis } \\
\text { fungoides) }\end{array}$ & 0.35 & $0 \cdot 34-0 \cdot 36$ & 5526 & 1790 & $81 \cdot 5 \%$ & $80 \cdot 0-83 \cdot 1$ & 5482 \\
\hline Other T-cell lymphomas and natural killer cell neoplasms & 0.62 & $0.60-0.63$ & 9656 & 3128 & $39 \cdot 0 \%$ & $37 \cdot 9-40 \cdot 2$ & 9635 \\
\hline Diffuse B-cell lymphoma & $4 \cdot 32$ & $4 \cdot 29-4 \cdot 35$ & 67645 & 21910 & $53 \cdot 4 \%$ & $52 \cdot 9-53 \cdot 9$ & 67907 \\
\hline Follicular B-cell lymphoma & $2 \cdot 19$ & $2 \cdot 17-2 \cdot 22$ & 34346 & 11125 & $77 \cdot 0 \%$ & $76 \cdot 4-77 \cdot 6$ & 34545 \\
\hline Hairy cell leukaemia & 0.28 & $0.27-0.29$ & 4375 & 1417 & $89 \cdot 8 \%$ & $88 \cdot 3-91 \cdot 3$ & 4387 \\
\hline Plasmacytoma or multiple myeloma (and heavy chain diseases) & 5.71 & $5 \cdot 67-5 \cdot 75$ & 89440 & 28970 & $35 \cdot 3 \%$ & $34 \cdot 8-35 \cdot 7$ & 86496 \\
\hline Mantle cell lymphoma & 0.56 & $0.55-0.57$ & 8748 & 2834 & $44 \cdot 0 \%$ & $42 \cdot 6-45 \cdot 4$ & 8797 \\
\hline Prolymphocytic leukaemia, B cell & 0.05 & $0.05-0.06$ & 804 & 260 & $30 \cdot 8 \%$ & $26 \cdot 9-35 \cdot 2$ & 788 \\
\hline Acute myeloid leukaemia and related precursor neoplasms & 3.81 & $3 \cdot 77-3 \cdot 84$ & 59608 & 21557 & $19 \cdot 2 \%$ & $18 \cdot 8-19 \cdot 6$ & 56709 \\
\hline Acute promyelocytic leukaemia with $\mathrm{t}(15 ; 17)$ with variants & 0.12 & $0 \cdot 11-0.13$ & 1876 & 608 & $63 \cdot 2 \%$ & $60 \cdot 8-65 \cdot 7$ & 1880 \\
\hline Acute myeloid leukaemia & $3 \cdot 50$ & $3 \cdot 47-3 \cdot 53$ & 54789 & 17746 & $17.5 \%$ & $17 \cdot 1-17 \cdot 8$ & 52305 \\
\hline Myeloproliferative neoplasms & $3 \cdot 31$ & $3 \cdot 28-3 \cdot 34$ & 51888 & 18805 & $68 \cdot 3 \%$ & $67 \cdot 7-68 \cdot 9$ & 50624 \\
\hline Chronic myeloid leukaemia & $1 \cdot 12$ & $1 \cdot 10-1 \cdot 13$ & 17473 & 5660 & $54.9 \%$ & $54 \cdot 0-55 \cdot 9$ & 16599 \\
\hline Other myeloproliferative neoplasms & $2 \cdot 17$ & $2 \cdot 14-2 \cdot 19$ & 33954 & 10998 & $75 \cdot 0 \%$ & $74 \cdot 3-75 \cdot 7$ & 33599 \\
\hline Mast cell tumour & 0.03 & $0.03-0.03$ & 461 & 149 & $71 \cdot 4 \%$ & $66 \cdot 2-77 \cdot 1$ & 454 \\
\hline $\begin{array}{l}\text { Myelodysplastic syndrome and myelodysplastic or } \\
\text { myeloproliferative diseases }\end{array}$ & $2 \cdot 47$ & $2 \cdot 45-2 \cdot 50$ & 38738 & 15116 & $31 \cdot 1 \%$ & $30 \cdot 5-31 \cdot 8$ & 37792 \\
\hline Myelodysplastic syndrome with $5 q$ syndrome & 0.01 & $0.01-0.01$ & 156 & 51 & $48.0 \%$ & $38 \cdot 3-60 \cdot 3$ & 178 \\
\hline Other myelodysplastic syndrome & $2 \cdot 14$ & $2 \cdot 12-2 \cdot 16$ & 33542 & 10864 & $32 \cdot 2 \%$ & $31 \cdot 5-32 \cdot 9$ & 32576 \\
\hline Chronic myelomonocytic leukaemia & 0.29 & $0 \cdot 28-0 \cdot 30$ & 4542 & 1471 & $21 \cdot 3 \%$ & $19 \cdot 8-23 \cdot 0$ & 4575 \\
\hline Atypical chronic myeloid leukaemia BCR/ABL negative & 0.02 & $0.01-0.02$ & 239 & 77 & $28 \cdot 2 \%$ & $21 \cdot 7-36 \cdot 5$ & 248 \\
\hline Histiocytic and dendritic cell neoplasms & 0.05 & $0.05-0.06$ & 828 & 278 & $59.9 \%$ & $56 \cdot 1-63 \cdot 9$ & 817 \\
\hline Histiocytic malignancies & 0.04 & $0.04-0.05$ & 656 & 212 & $63 \cdot 4 \%$ & $59 \cdot 4-67 \cdot 8$ & 645 \\
\hline Lymph-node accessory cell tumours & 0.01 & $0.01-0.01$ & 172 & 56 & $45 \cdot 6 \%$ & $37 \cdot 1-56 \cdot 0$ & 172 \\
\hline All rare tier 2 tumours & 114.99 & $114 \cdot 82-115 \cdot 16$ & 1801443 & 636753 & $48.5 \%$ & $48 \cdot 4-48 \cdot 6$ & 1751601 \\
\hline Common: digestive tumours & $91 \cdot 80$ & $91.65-91.95$ & 1438094 & 490051 & $41 \cdot 4 \%$ & $41 \cdot 3-45 \cdot 8$ & 1365575 \\
\hline Epithelial tumours of stomach & $17 \cdot 10$ & $17 \cdot 03-17 \cdot 16$ & 267832 & 92067 & $21 \cdot 2 \%$ & $21 \cdot 0-21 \cdot 4$ & 253439 \\
\hline Adenocarcinoma with variants of stomach & $14 \cdot 18$ & $14 \cdot 12-14 \cdot 24$ & 222145 & 71954 & $22 \cdot 7 \%$ & $22 \cdot 5-22 \cdot 9$ & 221604 \\
\hline Epithelial tumours of colon & 43.88 & $43 \cdot 77-43 \cdot 98$ & 687386 & 234319 & $54 \cdot 2 \%$ & $54 \cdot 0-54 \cdot 4$ & 664118 \\
\hline Adenocarcinoma with variants of colon & 38.85 & $38.75-38.95$ & 608637 & 197139 & $57 \cdot 9 \%$ & $57 \cdot 7-58 \cdot 0$ & 604459 \\
\hline Epithelial tumours of rectum & 17.98 & $17.92-18.05$ & 281697 & 95187 & $53 \cdot 8 \%$ & $53 \cdot 6-54 \cdot 1$ & 276024 \\
\hline Adenocarcinoma with variants of rectum & $16 \cdot 45$ & $16 \cdot 39-16 \cdot 52$ & 257723 & 83477 & $55 \cdot 8 \%$ & $55 \cdot 6-56 \cdot 1$ & 258469 \\
\hline Epithelial tumours of pancreas & 12.84 & $12 \cdot 79-12 \cdot 90$ & 201179 & 68478 & $4 \cdot 1 \%$ & $4 \cdot 0-4 \cdot 2$ & 182579 \\
\hline \multirow[t]{2}{*}{ Adenocarcinoma with variants of pancreas } & $7 \cdot 96$ & 7.92-8.01 & 124744 & 40405 & $4 \cdot 1 \%$ & $4 \cdot 0-4 \cdot 2$ & 119154 \\
\hline & & & & & & \multicolumn{2}{|c|}{ (Table 1 continues on next page) } \\
\hline
\end{tabular}




\begin{tabular}{|c|c|c|c|c|c|c|c|}
\hline & $\begin{array}{l}\text { Crude incidence } \\
\text { rate per } \\
100000 \text { people } \\
\text { per year }\end{array}$ & $95 \% \mathrm{Cl}$ & $\begin{array}{l}\text { Observed cases } \\
\text { in } 83 \text { cancer } \\
\text { registries in } \\
2000-07\end{array}$ & $\begin{array}{l}\text { Estimated } \\
\text { new cases in } \\
2013 \text { in EU28 }\end{array}$ & $\begin{array}{l}\text { 5-year } \\
\text { relative } \\
\text { survival (\%) }\end{array}$ & $95 \% \mathrm{Cl}$ & $\begin{array}{l}\text { Observed cases } \\
\text { in } 94 \text { cancer } \\
\text { registries in } \\
2000-07\end{array}$ \\
\hline \multicolumn{8}{|l|}{ (Continued from previous page) } \\
\hline Common: thoracic tumours & 53.02 & 52.91-53.14 & 830611 & 281332 & $10 \cdot 1 \%$ & $10 \cdot 0-10 \cdot 2$ & 779539 \\
\hline Epithelial tumour of lung & 53.02 & $52 \cdot 91-53 \cdot 14$ & 830611 & 281332 & $10 \cdot 1 \%$ & $10 \cdot 0-10 \cdot 2$ & 779539 \\
\hline Squamous cell carcinoma with variants of lung & $12 \cdot 31$ & $12 \cdot 25-12 \cdot 36$ & 192771 & 62439 & $13 \cdot 8 \%$ & $13 \cdot 6-14 \cdot 0$ & 190051 \\
\hline Adenocarcinoma with variants of lung & 11.63 & $11 \cdot 58-11 \cdot 68$ & 182175 & 59007 & $16.0 \%$ & $15 \cdot 8-16 \cdot 2$ & 179385 \\
\hline Poorly differentiated endocrine carcinoma of lung & $7 \cdot 91$ & $7 \cdot 86-7 \cdot 95$ & 123888 & 40128 & $5.9 \%$ & $5 \cdot 7-6 \cdot 0$ & 121904 \\
\hline Common: female genital tumours & $74 \cdot 17$ & $74 \cdot 03-74 \cdot 30$ & 1161864 & 394087 & $82 \cdot 2 \%$ & $82 \cdot 1-82 \cdot 3$ & 1131902 \\
\hline Epithelial tumours of breast & 63.52 & $63 \cdot 40-63 \cdot 65$ & 995119 & 318878 & $82 \cdot 4 \%$ & $82 \cdot 3-82 \cdot 5$ & 961378 \\
\hline Invasive ductal carcinoma of breast & $46 \cdot 56$ & $46 \cdot 45-46 \cdot 66$ & 729345 & 236237 & $85 \cdot 4 \%$ & $85 \cdot 3-85 \cdot 6$ & 723998 \\
\hline Invasive lobular carcinoma of breast & $7 \cdot 75$ & $7 \cdot 71-7 \cdot 80$ & 121455 & 39340 & $86 \cdot 2 \%$ & $85 \cdot 9-86 \cdot 5$ & 120973 \\
\hline Epithelial tumours of corpus uteri & $10 \cdot 64$ & $10 \cdot 59-10 \cdot 70$ & 166745 & 75209 & $81 \cdot 2 \%$ & $80 \cdot 9-81 \cdot 4$ & 164787 \\
\hline Adenocarcinoma with variants of corpus uteri & 9.93 & $9 \cdot 88-9.98$ & 155550 & 50383 & $83.0 \%$ & $82 \cdot 7-83 \cdot 2$ & 154968 \\
\hline Common: male genital and urogenital tumours & $85 \cdot 27$ & $85 \cdot 13-85 \cdot 42$ & 1335876 & 462665 & $75 \cdot 9 \%$ & $75 \cdot 8-76 \cdot 0$ & 1277743 \\
\hline Epithelial tumours of prostate & 55.06 & $54 \cdot 95-55 \cdot 18$ & 862576 & 301113 & $84.0 \%$ & $83 \cdot 8-84 \cdot 1$ & 842467 \\
\hline Adenocarcinoma with variants of prostate & 48.86 & $48 \cdot 75-48 \cdot 97$ & 765405 & 247917 & $88.1 \%$ & $88 \cdot 0-88 \cdot 3$ & 762360 \\
\hline Epithelial tumours of kidney & 12.66 & $12 \cdot 61-12 \cdot 72$ & 198402 & 65848 & $60 \cdot 5 \%$ & $60 \cdot 2-60 \cdot 7$ & 187324 \\
\hline Renal cell carcinoma with variants & $10 \cdot 08$ & $10 \cdot 03-10 \cdot 13$ & 157886 & 51140 & $68 \cdot 5 \%$ & $68 \cdot 2-68 \cdot 8$ & 153460 \\
\hline Epithelial tumours of bladder & $17 \cdot 55$ & $17 \cdot 48-17 \cdot 61$ & 274896 & 95704 & $60 \cdot 4 \%$ & $60 \cdot 1-60 \cdot 6$ & 266941 \\
\hline Transitional cell carcinoma of bladder & 15.68 & $15 \cdot 62-15 \cdot 74$ & 245681 & 79577 & $62.7 \%$ & $62 \cdot 4-63 \cdot 0$ & 243620 \\
\hline Common: skin tumours and non-cutaneous melanoma & 69.08 & $68 \cdot 95-69 \cdot 21$ & 1082244 & 350542 & $95 \cdot 6 \%$ & $95 \cdot 5-95 \cdot 7$ & 1048046 \\
\hline Malignant skin melanoma & 14.06 & $14 \cdot 00-14 \cdot 12$ & 220206 & 71325 & $83.8 \%$ & $83 \cdot 6-84 \cdot 1$ & 216317 \\
\hline Epithelial tumours of skin & 55.03 & $54 \cdot 91-55 \cdot 14$ & 862038 & 279217 & $98.8 \%$ & $98 \cdot 7-99 \cdot 0$ & 837895 \\
\hline Basal cell carcinoma of skin & $40 \cdot 75$ & $40 \cdot 65-40 \cdot 85$ & 638347 & 206763 & $101 \cdot 6 \%$ & $101 \cdot 5-101 \cdot 8$ & 634953 \\
\hline Squamous cell carcinoma with variants of skin & $14 \cdot 28$ & $14 \cdot 22-14 \cdot 34$ & 223691 & 72454 & $89.7 \%$ & $89 \cdot 4-90 \cdot 1$ & 221487 \\
\hline Common: haematological malignancies & 11.03 & $10 \cdot 98-11.08$ & 172794 & 58286 & $60 \cdot 5 \%$ & $60 \cdot 2-60 \cdot 8$ & 166040 \\
\hline Lymphoid diseases & 11.03 & 10.98-11.08 & 172794 & 58286 & $60 \cdot 5 \%$ & $60 \cdot 2-60 \cdot 8$ & 166040 \\
\hline Other non-Hodgkin, mature B-cell lymphoma & $6 \cdot 37$ & $6 \cdot 33-6 \cdot 41$ & 99729 & 32303 & $68.3 \%$ & $67 \cdot 8-68 \cdot 7$ & 97389 \\
\hline All common tumours & $384 \cdot 37$ & $384 \cdot 07-384 \cdot 78$ & 6021483 & 2036963 & $63 \cdot 4 \%$ & $63 \cdot 3-63 \cdot 4$ & 5633710 \\
\hline
\end{tabular}

genital and urological tumours, endocrine organ tumours, CNS tumours, and sarcomas had overall incidences from 4 to 8 per 100000 people per year. Rare skin cancers and non-cutaneous melanoma, and embryonal cancers were the families with the lowest incidences $(1.22$ and 0.34 per 100000$)$. Overall, rare cancers accounted for $24 \%$ of all cancers diagnosed in EU28 during 2000-07; the majority were solid cancers (76\%). For sex-specific rare cancers, we also provide sex-specific incidence (appendix p 4).

5 -year relative survival of all rare cancers was $48 \cdot 5 \%$ (95\% CI 48.4-48.6), compared with $63.4 \%$ (95\% CI 63.3-63.4) for all common cancers. Rare cancers also had lower survival within the families of digestive cancers (15.3\% [95\% CI 15.2-15.5] for rare cancers vs $41.4 \%$ [41.3-41.5] for common cancers), female genital cancers (57.7\% [57.5-57.9] vs $82 \cdot 2 \%$ [82.1-82.3]), male genital and urological cancers $(73.6 \%$ [73.3-74.0] vs $75.9 \%$ [75.8-76.0]), skin cancers $(70 \cdot 2 \%$ [69.3-71.1] vs $95 \cdot 6 \%$ [95\% CI 95.5-95.7]), and haematological tumours
$(50 \cdot 5 \%[50 \cdot 3-50 \cdot 7]$ vs $60 \cdot 5 \%[60 \cdot 2-60 \cdot 8])$. The only exception was the thoracic cancer family $(13.4 \%$ $[13 \cdot 1-13 \cdot 6]$ vs $10 \cdot 1 \%[10 \cdot 0-10 \cdot 2])$, for which common cancers included poorly differentiated endocrine carcinoma of the lung with a very poor prognosis $(5.9 \%$ [5.7-6.0] after 5 years. Families including only rare cancers had 5-year relative survival ranging from high, as for embryonal tumours $(78 \cdot 6 \%$ [95\% CI 77.4-79.8]) and endocrine organ tumours $(88 \cdot 1 \%[87 \cdot 8-88 \cdot 4])$, to intermediate, for sarcomas $(59 \cdot 5 \%$ [57.4-58.2]), neuroendocrine tumours $(53 \cdot 5 \%$ [53.0-54.1]), and head and neck tumours $(52 \cdot 1 \%[51 \cdot 8-52 \cdot 3])$, and low for CNS tumours $(21 \cdot 3 \%$ [21.0-21.6]).

Time trends of incidence and survival for rare cancers are in figures 1 and 2 . Cancers whose incidence variation fell outside the confidence interval shown in figure 1 are listed in table 2, with the age-standardised incidence estimates for 1999-2002 and 2003-07, the corresponding annual percentage change and 3 standard-error confidence intervals. Rare cancer dots in the plot seem to be 


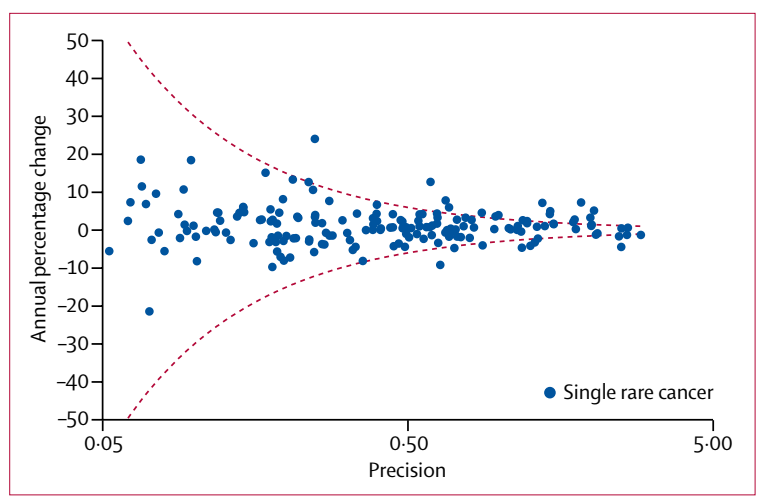

Figure 1: Annual percentage changes in age-adjusted incidence rates of rare cancers (1999-2007)

Funnel plot in which each dot represents a single rare cancer, the $y$-axis displays the estimated difference in terms of annual percentage change of age-adjusted incidence, and the $x$-axis the corresponding precision in terms of the inverse of its standard error. 3 standard-error confidence bounds for estimated zero changes ${ }^{7}$ are represented by two symmetrical lines progressively approaching the $x$-axis with increasing $x$ values. Dots lying above or below the area between them correspond respectively to tumours with $99.8 \%$ significantly higher or lower incidence rates.

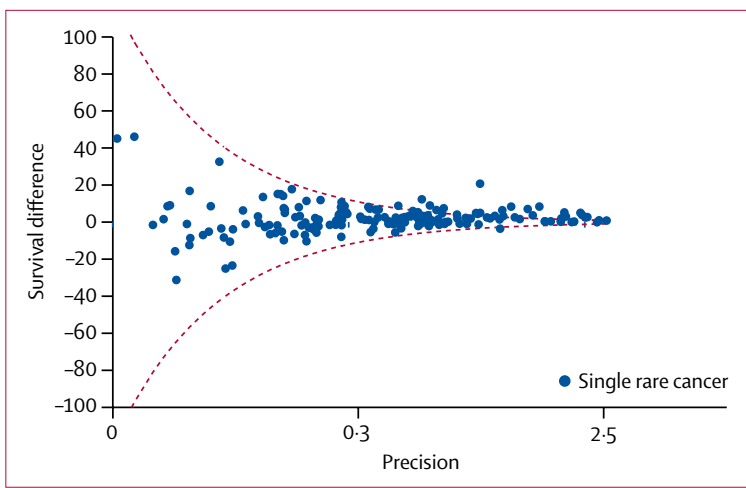

Figure 2: Difference in 5-year relative survival for rare cancers (1999-2001 vs 2005-07)

Funnel plot in which each dot represents a single rare cancer, the $y$-axis displays the estimated difference between 5-year relative survival in 2005-07 and 1999-2001, and the $x$-axis the corresponding precision in terms of the inverse of its standard error. 3 standard-error confidence bounds for estimated zero changes ${ }^{7}$ are represented by two symmetrical lines progressively approaching the $x$-axis with increasing $x$ values. Dots lying above or below the area between them correspond respectively to tumours with $99.8 \%$ significantly higher or lower incidence rates.

distributed fairly symmetrically around the zero-change line, indicating no major systematic shifts in incidence. The average annual percentage change of all rare cancers was $0 \cdot 5 \%(99 \cdot 8 \%$ CI $0 \cdot 3-0 \cdot 8)$. Incidence increased for 16 rare cancers and incidence decreased for 10 rare cancers, but with changes falling outside the $99.8 \%$ confidence limits. Time trends of rare cancers did not substantially differ from those of common cancers (data not shown), whose average annual change was $0 \cdot 9 \%$. Only prostate and skin cancers had an annual percentage change of more than $2 \%$, while only epithelial cancers of the stomach decreased more than $2 \%$ (data not shown).
Survival increased from 1999-2001 to 2005-07 for most rare cancers. The cloud of points in figure 2 is skewed upward from the zero line, corresponding to a mean increase in survival, averaged over all the entities, of $2 \cdot 9 \%(95 \%$ CI $2 \cdot 7-3 \cdot 2)$. Survival for 24 rare cancers was significantly increased (table 3), whereas survival was significantly reduced for only one cancer (other myelodysplastic syndromes). Rare cancers with the largest survival increases were mainly haematological: chronic myeloid leukaemia, diffuse B cell lymphoma, follicular lymphoma, precursor B/T cell lymphoblastic leukaemia or lymphoma, and multiple myeloma. Sarcomas were well represented among the top tumours with increasing survival, specifically of the viscera, trunk, and Kaposi's sarcoma. Survival increases higher than 5 percentage points were also observed for infiltrating ductal carcinoma of the prostate $(12 \cdot 3$ percentage points [99.8\% CI $6 \cdot 4-18 \cdot 2])$, poorly differentiated endocrine carcinoma of the digestive system $(7 \cdot 5$ percentage points $[2 \cdot 7-12 \cdot 2])$, and squamous cell carcinoma of the oropharynx $(7 \cdot 1$ percentage points $[5 \cdot 0-9 \cdot 2])$. There were no major improvements in survival for rare cancers of the colon, rectum, breast, or kidney, differently from the corresponding groups of common cancers. ${ }^{8}$

The extent of centralisation of rare cancer treatment is presented in figures 3 and 4 presenting mean admission volume, overall and by country, for 38 cancer groups ranked by decreasing incidence. We used a logarithmic scale for the $\mathrm{x}$-axis to make the graph readable despite large mean admission volume variability (from $0 \cdot 2$ to 82.6 treatments per year) across the considered cancers. The mean number of admissions is in the appendix (p 3-4). Pooled mean admission volume (figures 3 and 4) ranged from a maximum of 82.6 treatments per year for head and neck tumours to less than $0 \cdot 5$ treatments per year for placenta (choriocarcinoma), and some embryonal and endocrine tumours. The higher the incidence, the larger the mean admission volume of treating hospitals. The association between cancer incidence and mean admission volume in the pool of countries was very strong (Pearson's correlation coefficient $0 \cdot 88$ ), although there are several outliers. For example, epithelial tumours of the ovary had a higher incidence but a lower mean admission volume than CNS tumours (35 vs 20 cases treated per year). Treatment of patients with epithelial tumours of the ovary was thus spread among a larger number of hospitals compared with CNS tumours. Similarly, the incidence of soft tissue sarcomas was 5 times higher than bone sarcomas, but soft tissue sarcomas were treated centrally less than bone sarcoma. Treatment for thyroid cancers, uveal melanoma, and several embryonal tumours appeared to be fairly concentrated in a few hospitals with relatively high volumes. By contrast, tumours of the urinary tract, gastroenteropancreatic neuroendocrine tumours, small intestine, non-epithelial ovary cancers, and 


\begin{tabular}{|c|c|c|c|c|}
\hline & $\begin{array}{l}\text { 1999-2002 } \\
\text { age-standardised } \\
\text { incidence }\end{array}$ & $\begin{array}{l}2003-07 \\
\text { age-standardised } \\
\text { incidence }\end{array}$ & $\begin{array}{l}\text { Annual } \\
\text { percentage } \\
\text { change }\end{array}$ & $99.8 \% \mathrm{Cl}$ \\
\hline Gastrointestinal stromal sarcoma & 0.098 & 0.258 & $24 \cdot 1 \%$ & $12 \cdot 0$ to $36 \cdot 2$ \\
\hline $\begin{array}{l}\text { Gastroenteric-pancreatic tumour, poorly differentiated endocrine } \\
\text { carcinoma of pancreas and digestive system }\end{array}$ & 0.361 & 0.618 & $12 \cdot 7 \%$ & $7 \cdot 7$ to $17 \cdot 8$ \\
\hline Other T-cell lymphomas and natural killer cell neoplasms & 0.395 & 0.555 & $7.8 \%$ & 3.3 to 12.4 \\
\hline Diffuse B-cell lymphoma & 2.837 & 3.894 & $7 \cdot 3 \%$ & 5.7 to 8.9 \\
\hline Other myeloproliferative neoplasms & 1.530 & 2.092 & $7 \cdot 2 \%$ & 5.0 to 9.4 \\
\hline Mantle cell lymphoma & 0.367 & 0.477 & $6.0 \%$ & 1.6 to 10.4 \\
\hline Carcinomas of thyroid gland & 3.470 & $4 \cdot 353$ & $5 \cdot 2 \%$ & 3.7 to 6.6 \\
\hline Other myelodysplastic syndrome & $1 \cdot 395$ & $1 \cdot 738$ & $5.0 \%$ & 3.0 to 7.1 \\
\hline Squamous cell carcinoma with variants of anal canal & 0.595 & 0.728 & $4.6 \%$ & $1 \cdot 2$ to 8.0 \\
\hline Follicular B-cell lymphoma & 1.676 & $2 \cdot 021$ & $4 \cdot 2 \%$ & $2 \cdot 2$ to $6 \cdot 3$ \\
\hline Cholangiocarcinoma of intrahepatic bile tract & 0.685 & 0.816 & $4.0 \%$ & 0.9 to 7.0 \\
\hline Neuroendocrine carcinoma of other sites & 0.683 & 0.801 & $3.6 \%$ & 0.5 to 6.7 \\
\hline Adenocarcinoma with variants of oesophagus & 2.725 & 3.153 & $3.3 \%$ & $1 \cdot 8$ to 4.8 \\
\hline Squamous cell carcinoma with variants of oropharynx & $2 \cdot 412$ & 2.732 & $2 \cdot 8 \%$ & $1 \cdot 1$ to 4.5 \\
\hline Adenocarcinoma with variants of extrahepatic biliary tract & 0.969 & 1.088 & 2.6 & 0.1 to 5.1 \\
\hline Hepatocellular carcinoma of liver and intrahepatic bile tract & 2.068 & $2 \cdot 273$ & $2 \cdot 1 \%$ & 0.4 to 3.8 \\
\hline Squamous cell carcinoma with variants of cervix uteri & 4.536 & $4 \cdot 287$ & $-1 \cdot 2 \%$ & $-2 \cdot 4$ to $-0 \cdot 1$ \\
\hline Adenocarcinoma with variants of ovary & 5.351 & 5.053 & $-1 \cdot 3 \%$ & $-2 \cdot 3$ to -0.2 \\
\hline Squamous cell carcinoma with variants of larynx & 3.853 & 3.578 & $-1.6 \%$ & -2.8 to -0.4 \\
\hline Chronic myeloid leukaemia & 0.991 & 0.854 & $-3 \cdot 2 \%$ & -5.5 to -0.9 \\
\hline Infiltrating duct carcinoma of prostate & 0.412 & 0.343 & $-4 \cdot 0 \%$ & -7.4 to -0.6 \\
\hline Squamous cell carcinoma with variants of lip & 0.838 & 0.693 & $-4 \cdot 1 \%$ & -6.5 to -1.8 \\
\hline Large cell carcinoma of lung & 3.440 & 2.806 & $-4 \cdot 4 \%$ & $-5 \cdot 6$ to $-3 \cdot 2$ \\
\hline Mucinous adenocarcinoma of ovary & 0.813 & 0.657 & $-4 \cdot 6 \%$ & $-7 \cdot 2$ to $-2 \cdot 1$ \\
\hline Adenocarcinoma with variants of bladder & 0.265 & 0.213 & $-4 \cdot 7 \%$ & -8.9 to -0.5 \\
\hline Undifferentiated carcinoma of stomach & 0.189 & 0.123 & $-9 \cdot 2 \%$ & -13.9 to -4.5 \\
\hline
\end{tabular}

neuroendocrine tumours of the skin were treated in centres with an even lower mean admission volume than would be expected because of their very low occurrence.

With some exceptions, country-specific patterns of mean admission volume were similar to the pooled data. By contrast with what was found in the other countries, the management of epithelial ovarian cancers was highly centralised in Bulgaria and Slovenia. Patients with CNS tumours were treated in highly centralised structures in all countries except Finland and Navarra. Treatment for uveal melanoma and retinoblastoma was not centralised in Bulgaria or in Navarra. Slovenia and the Netherlands had the highest centralisation patterns, whereas mean admission volume for the majority of cancers was very low in Navarra due also to the small population size.

Table 4 shows the annual number of cases diagnosed, the number of top-volume hospitals treating at least $75 \%$ of national cases, and the average annual numbers of treatments provided for each country and for 29 rare cancers. For head and neck cancers, $75 \%$ of patients were centralised in two top hospitals in Slovenia ( 2 million population, 266 treatments per hospital per year), and 12 top hospitals in the Netherlands (17 million population, 201 treatments per hospital per year). The level of centralisation was lower in the other countries, resulting in a caseload of 145 in the ten Bulgarian top hospitals, 106 in the 29 Belgian hospitals, and 83 in the six hospitals in Finland, 77 in the two hospitals in Navarra, and 63 in the seven hospitals in Ireland. The Netherlands and Slovenia had the highest treatment volume for 12 of 29 cancers.

\section{Discussion}

Rare cancers make up one quarter of all malignancies. They are a very heterogeneous group of almost 200 cancers, mostly solid, constituting from $2 \%$ of all skin cancers up to $32 \%$ of all female genital cancers. We confirmed that 5-year survival is lower for rare cancers than common cancers (49\% vs $63 \%$ ), and for all rare cancer families compared with their more common counterparts, except thoracic cancers. The disadvantage persisted even after excluding common cancers with good prognosis, such as prostate, breast, and skin cancer. Several factors help explain these differences: the biology 


\begin{tabular}{|c|c|c|c|c|}
\hline & $\begin{array}{l}1999-2001 \\
5 \text {-year } \\
\text { relative } \\
\text { survival }\end{array}$ & $\begin{array}{l}2005-07 \\
5 \text {-year } \\
\text { relative } \\
\text { survival }\end{array}$ & Difference & $99.8 \% \mathrm{Cl}$ \\
\hline Chronic myeloid leukaemia & $37 \cdot 2$ & $57 \cdot 9$ & $20 \cdot 7$ & $17 \cdot 4$ to $24 \cdot 1$ \\
\hline Infiltrating duct carcinoma of prostate & $67 \cdot 5$ & $79 \cdot 8$ & $12 \cdot 3$ & $6 \cdot 4$ to $18 \cdot 2$ \\
\hline Soft tissue sarcoma of viscera & $34 \cdot 7$ & $43 \cdot 7$ & $9 \cdot 0$ & 3.6 to $14 \cdot 4$ \\
\hline Kaposi's sarcoma & $75 \cdot 4$ & $84 \cdot 2$ & 8.8 & $1 \cdot 4$ to $16 \cdot 2$ \\
\hline Diffuse B-cell lymphoma & $46 \cdot 9$ & $55 \cdot 2$ & 8.4 & $6 \cdot 5$ to $10 \cdot 2$ \\
\hline Follicular B-cell lymphoma & $69 \cdot 5$ & $77 \cdot 9$ & 8.4 & $5 \cdot 9$ to $10 \cdot 8$ \\
\hline $\begin{array}{l}\text { Poorly differentiated endocrine carcinoma of } \\
\text { pancreas and digestive system }\end{array}$ & $25 \cdot 3$ & $32 \cdot 7$ & $7 \cdot 5$ & $2 \cdot 7$ to $12 \cdot 2$ \\
\hline Squamous cell carcinoma with variants of oropharynx & $37 \cdot 5$ & $44 \cdot 5$ & $7 \cdot 1$ & $5 \cdot 0$ to $9 \cdot 2$ \\
\hline Soft tissue sarcoma of superficial trunk & $43 \cdot 9$ & $50 \cdot 4$ & $6 \cdot 5$ & 1.4 to 11.6 \\
\hline $\begin{array}{l}\text { Precursor B/T cell lymphoblastic leukaemia or } \\
\text { lymphoma (and Burkitt's leukaemia/lymphoma) }\end{array}$ & $54 \cdot 3$ & $60 \cdot 8$ & $6 \cdot 4$ & 3.8 to 9.1 \\
\hline $\begin{array}{l}\text { Plasmacytoma or multiple myeloma (and heavy chain } \\
\text { diseases) }\end{array}$ & $29 \cdot 8$ & $35 \cdot 0$ & $5 \cdot 2$ & 3.8 to 6.7 \\
\hline Carcinomas of thyroid gland & $85 \cdot 6$ & $90 \cdot 6$ & $5 \cdot 0$ & $3 \cdot 8$ to $6 \cdot 3$ \\
\hline Adenocarcinoma with variants of cervix uteri & $63 \cdot 8$ & $68 \cdot 8$ & $5 \cdot 0$ & $1 \cdot 7$ to $8 \cdot 3$ \\
\hline $\begin{array}{l}\text { Well differentiated not functioning endocrine } \\
\text { carcinoma of pancreas and digestive system }\end{array}$ & $67 \cdot 7$ & $72 \cdot 6$ & $4 \cdot 9$ & 1.5 to 8.4 \\
\hline Soft tissue sarcoma of limbs & 63.9 & $68 \cdot 4$ & $4 \cdot 4$ & 1.0 to 7.9 \\
\hline Adenocarcinoma with variants of oesophagus & $9 \cdot 9$ & $13 \cdot 8$ & 3.9 & $2 \cdot 6$ to $5 \cdot 1$ \\
\hline Squamous cell carcinoma with variants of oral cavity & $46 \cdot 1$ & $49 \cdot 7$ & $3 \cdot 7$ & 1.7 to 5.6 \\
\hline Squamous cell carcinoma with variants of hypopharynx & $22 \cdot 2$ & $25 \cdot 6$ & $3 \cdot 4$ & $0 \cdot 5$ to $6 \cdot 3$ \\
\hline Other myeloproliferative neoplasms & $70 \cdot 8$ & $74 \cdot 0$ & $3 \cdot 2$ & 0.6 to 5.9 \\
\hline Squamous cell carcinoma with variants of cervix uteri & $65 \cdot 1$ & $68 \cdot 1$ & $3 \cdot 0$ & 1.6 to 4.5 \\
\hline Large cell carcinoma of lung & $10 \cdot 9$ & $13 \cdot 6$ & $2 \cdot 7$ & 1.6 to 3.9 \\
\hline $\begin{array}{l}\text { Adenocarcinoma with variants of extrahepatic biliary } \\
\text { tract }\end{array}$ & $16 \cdot 2$ & $18 \cdot 7$ & $2 \cdot 6$ & 0.2 to $5 \cdot 0$ \\
\hline Squamous cell carcinoma with variants of oesophagus & $9 \cdot 5$ & $12 \cdot 0$ & 2.5 & $1 \cdot 3$ to $3 \cdot 7$ \\
\hline $\begin{array}{l}\text { Hepatocellular carcinoma of liver and intrahepatic } \\
\text { biliary tract }\end{array}$ & $11 \cdot 0$ & $13 \cdot 0$ & $2 \cdot 0$ & 0.5 to 3.5 \\
\hline Other myelodysplastic syndrome & $33 \cdot 8$ & $30 \cdot 2$ & $-3 \cdot 5$ & $-6 \cdot 3$ to -0.8 \\
\hline \multicolumn{5}{|c|}{ Data are rare cancers lying outside the 3 standard-errors confidence bounds in figure 2 . } \\
\hline
\end{tabular}

of the diseases, adequacies of diagnosis and treatment, scarcity of effective therapies, or insufficient evidencebased treatment guidelines.

A novelty of this study is the analysis of incidence and survival trends. Overall, incidence rose by $0.5 \%$ a year from 1999 up to 2007. The increase was substantial for several rare cancers. Some of the increases can probably be attributed to improvements in pathological diagnosis, new entity codes in the ICD-O-3, and to the time needed to adapt the coding procedures. This is the case for gastrointestinal stromal tumours, large cell carcinomas of the lung, neuroendocrine tumours, and many haematological cancers. ${ }^{9-11}$ For other rare cancers, increases in incidence might be due to improvements in pathological diagnosis, similar to neuroendocrine tumours. For thyroid carcinoma several authors have suggested an increase in overdiagnosis. ${ }^{12}$ However, increased exposure to risk factors might explain higher incidence rates for some cancers; such as exposure to HPV for oropharynx and anal canal squamous cell cancers ${ }^{13,14}$ and perhaps increasing obesity or gastrooesophageal reflux for adenocarcinoma of the oesophagus. ${ }^{15}$ The low incidence of squamous cell carcinoma of the cervix might reflect organised cervical screening programmes. The drop in incidence for some of the rare cancers was due to the still falling prevalence of smoking. ${ }^{16}$

Relative survival improved by about $3 \%$ overall over the study period, slightly less than for common cancers $(5 \cdot 5 \%$, data not shown), suggesting that investments were more focused on common cancers. Also, overdiagnosis is expected to affect more common cancers than rare cancers. Improvement to relative survival was greatest for chronic myeloid leukaemia with a 5-year gain in survival of $21 \%$ across the study years, largely explained by the widespread use of new and more effective treatments, such as targeted treatments and more effective stem-cell transplantation. ${ }^{17}$ For many other haematological cancers, new (targeted) drugs in combination with radiotherapy, and improvements in transplantation are responsible for the effects on prognosis. ${ }^{18}$ Survival also improved for some groups of sarcoma (viscera, trunk, and limbs) for which multidisciplinary approaches and centralisation of treatments might take the credit; similarly, for neuroendocrine tumours, ${ }^{19}$ biliary tract, liver, ${ }^{20}$ and oesophageal cancers, ${ }^{15}$ for which there are now more specific and effective treatments and protocols. For oesophageal cancers, earlier detection through Barrett's oesophagus surveillance practices might also contribute to improvements in relative survival. For oropharyngeal cancers, the larger proportion of less aggressive tumours attributed to HPV might have influenced the survival gain. ${ }^{21}$ For carcinoma of the thyroid and infiltrating ductal carcinoma of the prostate, early diagnosis is probably the major contributing factor to improved relative survival. Early diagnosis would also have contributed to a rise in the proportion of cases that are clinically irrelevant, although this is hard to estimate. ${ }^{12,22}$ As for incidence, some of the apparent survival gains might be due to classification changes, ${ }^{9}$ such as for large cell carcinomas of the lung that have a relatively new ICD-O morphology code.

Myeloproliferative neoplasms and myelodysplastic syndromes were not considered cancers until the WHO classification was changed in 2001, and their registration started even later. ${ }^{9}$ Generally, the increases in incidence of some rare cancers could be due to more specific diagnosis and coding by cancer registry.

Our hospital volume analysis represents, to our knowledge, the first attempt to systematically study the place of treatment of rare cancers from population-based cancer registry data. Many potentially relevant indications

Figure 3: Mean admission volume by country and cancers ranked by pooled incidence leve Data are for pooled countries, Belgium, Bulgaria, and Finland. 


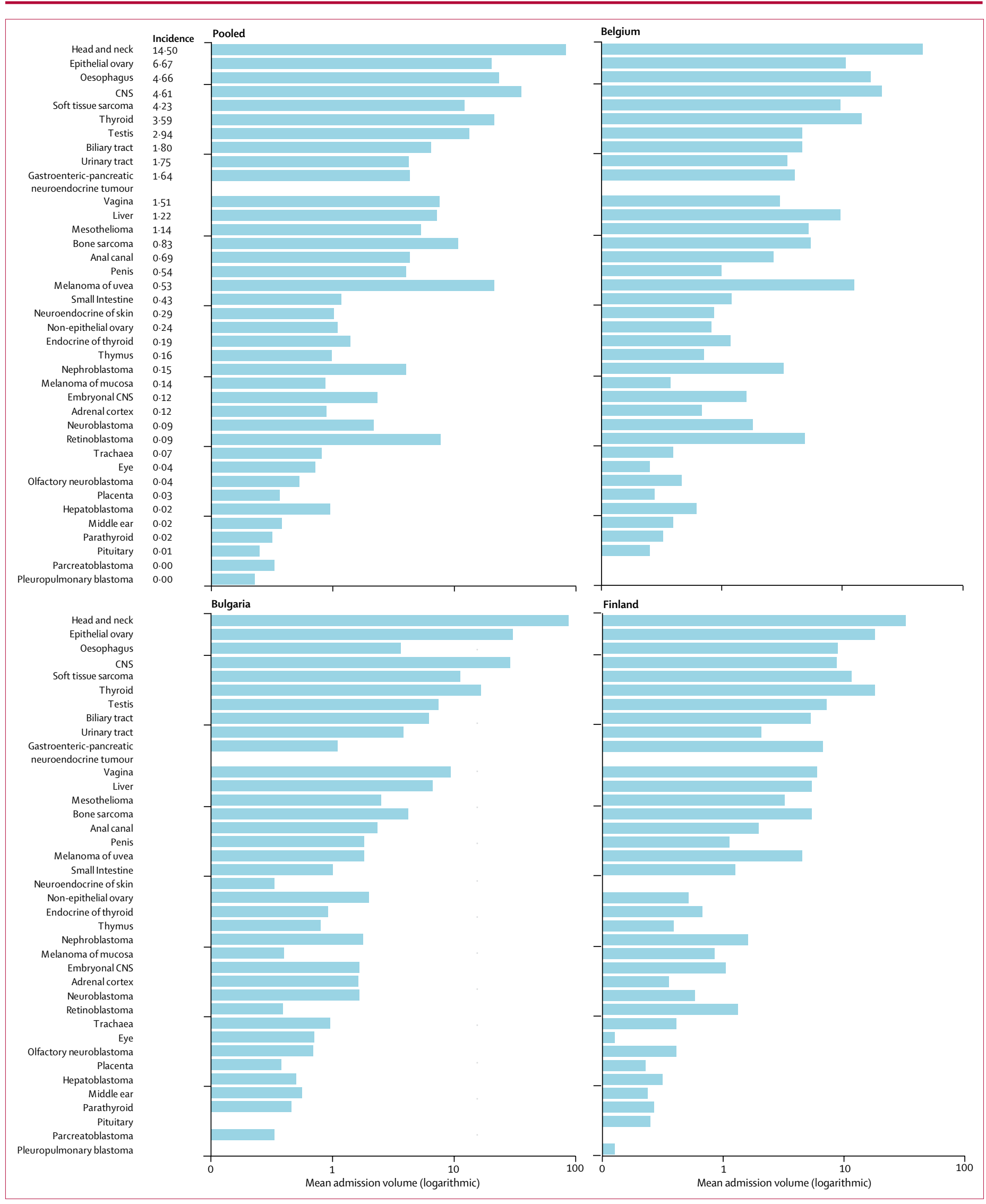


Articles

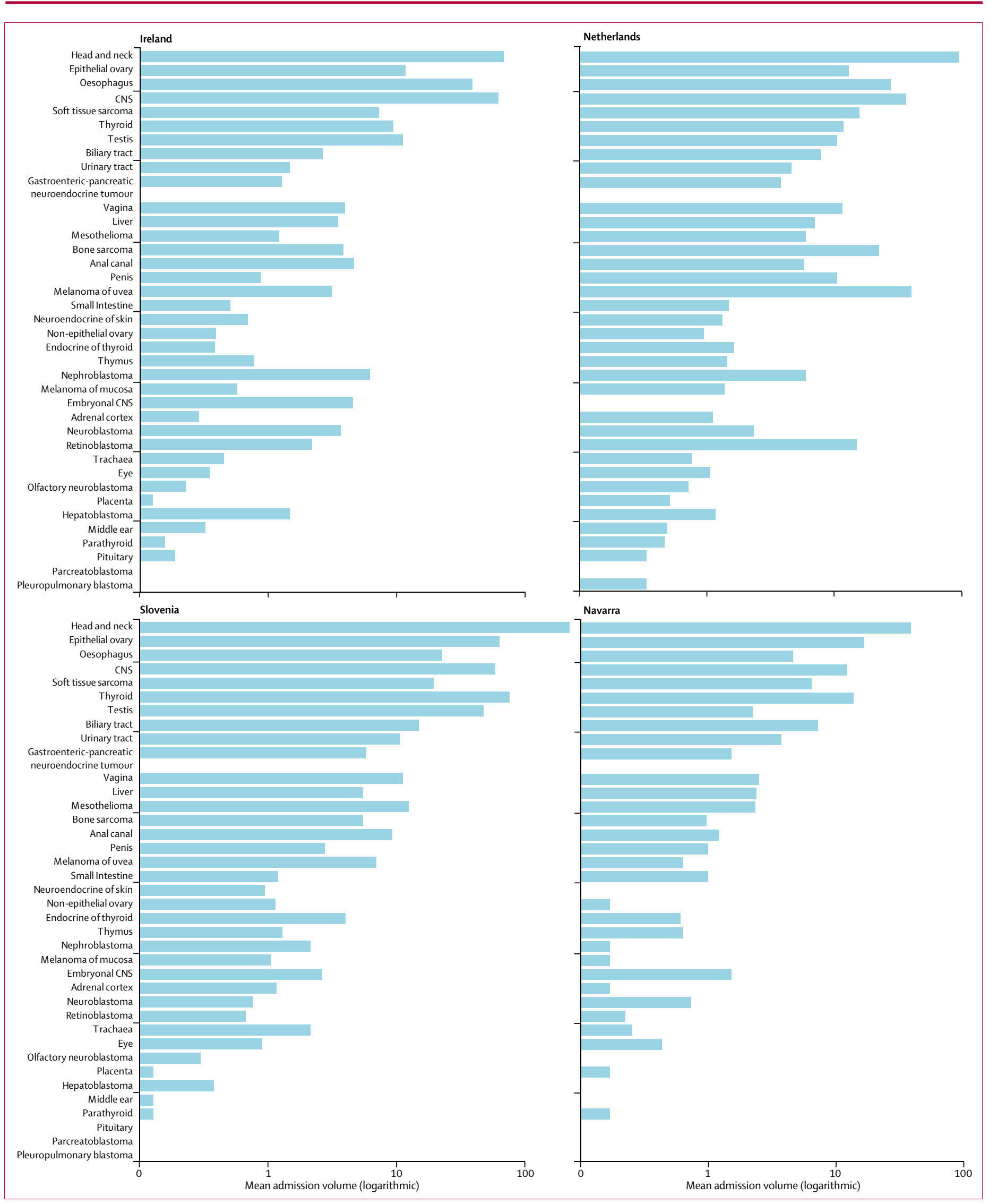


can be drawn from this seldom used source of information. However, several important limitations should be recognised. Seven cancer registries cannot be considered as statistically representative of the whole European population. Bulgaria, Finland, and Navarra only provided information about, at most, three treatments: the first surgical, systemic, and radiotherapy treatments. However, we estimated from the data of the other cancer registries that this problem only relates to about $1 \%$ of all patients.

The mean admission volume estimates, based on individual patient data and blind administrative coding of hospitals, will depend on how cancer services were organised and coded. We cannot know if, for some rare cancers and in some countries, hospitals were linked in organised networks during the study period, thus overcoming an apparent dispersion of treating structures. For example, patients with localised sarcomas or head and neck cancers were frequently treated by small or peripheral hospitals. ${ }^{23}$ If several hospitals provided different services but acted cooperatively as a single specialist centre, their estimated volume will depend on whether they were identified as single or separate units. Our data do not allow detailed identification of protocols used in the considered hospitals. Hospital volume can therefore be considered as only a partial quality indicator, mainly pointing to level of experience in protocol application and general management of patients with rare cancer.

To address the volume-survival assocation is beyond the scope of this paper, but centralisation of care has been suggested to improve outcomes for rare cancers. ${ }^{24}$ This is particularly true when optimal treatment requires complex surgery or high-technology radiotherapy equipment. Diagnosis and treatment in reference centres are expected to be more accurate because they benefit from large numbers of cases, which are often discussed in a multidisciplinary setting involving expert professionals. Often centralised sites are connected to research centres participating in international debates and research. Disadvantages of centralisation are the need for patients to locate to the centralised site, and the risk of a longer waiting list, with consequent discomfort and possible negative effects on outcome. ${ }^{25}$ Sometimes centralisation was only moderately perceived by oncologists as a solution to be endorsed for patients with rare cancers. ${ }^{26}$

For many of the solid rare cancers, centralisation did not seem to have been completely achieved during the study period. However, most cases had been diagnosed more than 10 years ago when centralisation for patients with cancer did not necessarily have much priority. Centralisation seemed to be more widely implemented for rare cancers requiring highly specific technologies (particularly radiotherapy and nuclear medicine) and for

Figure 4: Mean admission volume by country and cancers ranked by pooled incidence level

Data are for Ireland, The Netherlands, Slovenia, and Navarra. those with long-established evidence-based guidelines for diagnosis and treatment. This was the case for many paediatric tumours, uveal melanoma, anal canal cancers, adrenal cortex cancers and, for specific surgical expertise, in CNS cancers and bone sarcomas.

The degree of centralisation varied across Europe, and to a large extent was affected by the population size. In countries with a small population it is easier to concentrate patients in a single hospital or few hospitals. High admission volumes are more likely to be achieved in reference centres in larger-population countries.

The results of this part of the study were discussed in the participating countries at dedicated meetings attended by public health planners, oncologists, surgeons, representatives of Ministries of Health, and patient associations. Although the general pattern of dispersion was recognised, almost all the countries were working at different levels to implement centralisation, or networkbased organisations for treatment, or both, while still following country-specific priorities. ${ }^{27}$

In Belgium, where all patients with cancer can be treated in any hospital with an oncology care programme, the level of centralisation was low. A plan is now under way for the development of hospital networks between centres of expertise and other oncology care services and programmes. Centralisation was already happening while we did this study in the Netherlands, mostly for surgical treatment. This was reflected in the high admission volumes in this country for many rare cancers (see appendix p 3).

In Bulgaria, patients with rare cancers were operated in all hospitals with surgical departments, whereas radiotherapy was concentrated in 17 centres and systemic therapy in 14 oncological hospitals. A problem was the quality of diagnosis, mainly due to inadequate facilities to diagnose many complex rare cancers. The definition of national and international pathways for second opinions from expert pathologists was deemed important by participants, for improving quality of rare cancer diagnoses. With this in mind, the European Reference Networks should offer a good opportunity to improve pathologist training through dedicated training schemes and fellowships across Europe. Cancer registration remains vital for monitoring progress in rare cancer diagnosis and treatment for these patients.

In Finland, more than $60 \%$ of patients with rare cancer were treated in five university hospitals. Centralisation in single national structures was only observed for uveal melanoma and retinoblastoma. Further centralisation for other rare cancers is impeded by the spread of the population over large areas and by administrative constraints on regional health authorities for referring patients with cancer to the closest university hospital.

Irish public health authorities, during the period covered by the study, identified centres to treat rare or particularly complex cancers. However, patients were not always correctly referred to them. This highlights the 


\begin{tabular}{|c|c|c|c|c|c|c|c|c|c|c|c|c|c|c|c|c|c|c|c|c|c|}
\hline & \multicolumn{3}{|c|}{ Belgium $\left(10 \cdot 5^{\star}\right)$} & \multicolumn{3}{|c|}{ Bulgaria $\left(7 \cdot 7^{*}\right)$} & \multicolumn{3}{|c|}{ Finland $\left(5 \cdot 3^{*}\right)$} & \multicolumn{3}{|c|}{ Ireland $\left(4 \cdot 2^{*}\right)$} & \multicolumn{3}{|c|}{ Netherlands $\left(16 \cdot 3^{*}\right)$} & \multicolumn{3}{|c|}{ Slovenia $\left(2 \cdot 0^{\star}\right)$} & \multicolumn{3}{|c|}{ Navarra $\left(0.6^{*}\right)$} \\
\hline & Cases & $\mathrm{H} 75$ & Treat & Cases & H75 & Treat & Cases & H75 & Treat & Cases & $\mathrm{H} 75$ & Treat & Cases & $\mathrm{H} 75$ & Treat & Cases & $\mathrm{H} 75$ & Treat & Cases & $\mathrm{H} 75$ & Treat \\
\hline Head and neck & 2098 & 29 & 105.6 & 1180 & 10 & $145 \cdot 1$ & 439 & 6 & $82 \cdot 2$ & 368 & 7 & 63.0 & 2439 & 12 & $201 \cdot 4$ & 395 & 2 & $266 \cdot 1$ & 125 & 2 & $76 \cdot 6$ \\
\hline Epithelial ovary & 760 & 50 & $19 \cdot 5$ & 627 & 16 & $52 \cdot 3$ & 370 & 10 & $44 \cdot 5$ & 261 & 15 & $21 \cdot 0$ & 1118 & 47 & $30 \cdot 2$ & 158 & 3 & $82 \cdot 0$ & 38 & 1 & $45 \cdot 5$ \\
\hline Oesophagus & 689 & 31 & $29 \cdot 3$ & 77 & 14 & $5 \cdot 2$ & 163 & 8 & 21.6 & 289 & 9 & 37.1 & 1422 & 31 & $42 \cdot 0$ & 49 & 2 & 32.9 & 24 & 2 & $15 \cdot 7$ \\
\hline CNS & 623 & 20 & $48 \cdot 4$ & 412 & 13 & $41 \cdot 7$ & 57 & 4 & $19 \cdot 1$ & 229 & 3 & $106 \cdot 3$ & 912 & 14 & $84 \cdot 0$ & 97 & 2 & 78.7 & 47 & 2 & $32 \cdot 0$ \\
\hline Soft tissue sarcoma & 500 & 35 & 16.6 & 372 & 21 & $18 \cdot 4$ & 165 & 7 & $25 \cdot 6$ & 157 & 17 & 10.6 & 802 & 33 & $26 \cdot 4$ & 81 & 2 & $47 \cdot 4$ & 32 & 2 & $17 \cdot 4$ \\
\hline Thyroid & 576 & 34 & $14 \cdot 2$ & 220 & 12 & $20 \cdot 4$ & 286 & 12 & 22.8 & 98 & 11 & 9.6 & 418 & 31 & $17 \cdot 1$ & 109 & 1 & $260 \cdot 3$ & 43 & 2 & 36.8 \\
\hline Testis & 244 & 40 & 8.4 & 180 & 19 & $12 \cdot 4$ & 101 & 9 & $14 \cdot 3$ & 144 & 12 & $15 \cdot 6$ & 609 & 42 & $18 \cdot 4$ & 93 & 3 & 48.8 & 10 & 3 & $4 \cdot 4$ \\
\hline Biliary tract & 214 & 44 & $4 \cdot 9$ & 183 & 23 & 6.5 & 147 & 13 & $11 \cdot 3$ & 122 & 14 & $7 \cdot 7$ & 582 & 38 & $12 \cdot 2$ & 47 & 3 & $13 \cdot 2$ & 43 & 2 & $19 \cdot 7$ \\
\hline $\begin{array}{l}\text { Gastroenteric-pancreatic } \\
\text { neuroendocrine tumour }\end{array}$ & 287 & 46 & $5 \cdot 6$ & 30 & 21 & $1 \cdot 3$ & 148 & 13 & $9 \cdot 3$ & 61 & 20 & $2 \cdot 7$ & 355 & 44 & $6 \cdot 9$ & 22 & 3 & 6.8 & 10 & 3 & 2.9 \\
\hline Liver & 250 & 22 & $11 \cdot 0$ & 107 & 12 & $7 \cdot 6$ & 165 & 11 & $12 \cdot 8$ & 68 & 12 & 4.6 & 236 & 36 & $5 \cdot 2$ & 29 & 2 & $14 \cdot 4$ & 49 & 3 & $14 \cdot 5$ \\
\hline Urinary tract & 292 & 48 & $6 \cdot 7$ & 67 & 17 & $4 \cdot 1$ & 48 & 12 & 3.9 & 24 & 10 & $2 \cdot 3$ & 419 & 46 & $7 \cdot 7$ & 30 & 3 & 8.9 & 19 & 3 & 8.2 \\
\hline Mesothelioma & 184 & 25 & 8.7 & 34 & 10 & $3 \cdot 7$ & 64 & 9 & 6.8 & 25 & 11 & $2 \cdot 0$ & 481 & 43 & 9.8 & 21 & 1 & $22 \cdot 3$ & 9 & 2 & 4.6 \\
\hline Vagina & 172 & 35 & $5 \cdot 8$ & 120 & 9 & $14 \cdot 0$ & 70 & 5 & 14.8 & 40 & 9 & $4 \cdot 7$ & 296 & 14 & 21.8 & 42 & 2 & 21.9 & 8 & 2 & 4.7 \\
\hline Bone sarcoma & 81 & 10 & $10 \cdot 2$ & 55 & 13 & 4.6 & 28 & 3 & 9.6 & 30 & 7 & $5 \cdot 2$ & 195 & 5 & $43 \cdot 3$ & 15 & 2 & $10 \cdot 4$ & 3 & 2 & $2 \cdot 4$ \\
\hline Anal canal & 95 & 27 & $5 \cdot 3$ & 39 & 12 & $4 \cdot 1$ & 24 & 7 & $4 \cdot 6$ & 30 & 9 & $4 \cdot 4$ & 135 & 22 & $7 \cdot 2$ & 15 & 1 & $23 \cdot 6$ & 4 & 2 & 3.6 \\
\hline Melanoma of uvea & 43 & 2 & $21 \cdot 9$ & 17 & 7 & $2 \cdot 7$ & 6 & 1 & 5.5 & 29 & 4 & $5 \cdot 7$ & 156 & 2 & $80 \cdot 2$ & 13 & 1 & 11.9 & 3 & 3 & 0.8 \\
\hline Penis & 63 & 43 & $1 \cdot 4$ & 39 & 17 & $2 \cdot 4$ & 21 & 10 & $2 \cdot 1$ & 20 & 15 & $1 \cdot 2$ & 109 & 26 & $3 \cdot 7$ & 9 & 4 & $2 \cdot 0$ & 4 & 3 & 1.2 \\
\hline Small intestine & 62 & 37 & 1.9 & 15 & 13 & $1 \cdot 1$ & 26 & 13 & $2 \cdot 1$ & 27 & 20 & $1 \cdot 3$ & 120 & 38 & 2.6 & 5 & 4 & $1 \cdot 3$ & 2 & 2 & 1.0 \\
\hline $\begin{array}{l}\text { Neuroendocrine } \\
\text { carcinoma of skin }\end{array}$ & 46 & 32 & 1.9 & 1 & 3 & 0.4 & 0 & & & 15 & 18 & 0.8 & 77 & 37 & $2 \cdot 3$ & 4 & 4 & $1 \cdot 1$ & 0 & & \\
\hline Non-epithelial ovary & 20 & 19 & $1 \cdot 3$ & 43 & 17 & $3 \cdot 2$ & 8 & 9 & $1 \cdot 1$ & 8 & 15 & 0.6 & 32 & 24 & 1.4 & 4 & 3 & 1.7 & 1 & 3 & 0.3 \\
\hline $\begin{array}{l}\text { Endocrine carcinoma of } \\
\text { thyroid }\end{array}$ & 31 & 22 & $1 \cdot 4$ & 10 & 9 & $1 \cdot 2$ & 8 & 8 & $1 \cdot 2$ & 5 & 10 & 0.5 & 32 & 13 & $2 \cdot 7$ & 5 & 1 & $10 \cdot 3$ & 1 & 1 & 1.7 \\
\hline Thymus & 22 & 20 & 1.4 & 7 & 8 & $1 \cdot 3$ & 4 & 5 & $1 \cdot 1$ & 5 & 5 & $1 \cdot 3$ & 36 & 15 & $2 \cdot 8$ & 3 & 2 & $2 \cdot 1$ & 2 & 2 & $1 \cdot 3$ \\
\hline Nephroblastoma & 18 & 4 & $7 \cdot 4$ & 6 & 3 & 2.8 & 8 & 3 & 4.7 & 7 & 1 & $13 \cdot 4$ & 30 & 4 & 16.9 & 3 & 1 & 4.8 & 0 & 1 & 0.3 \\
\hline Melanoma of mucosa & 14 & 24 & 0.8 & 2 & 5 & 0.8 & 10 & 7 & $1 \cdot 7$ & 6 & 11 & 0.6 & 34 & 13 & $3 \cdot 0$ & 4 & 3 & 1.5 & 1 & 2 & 0.3 \\
\hline Adrenal cortex & 13 & 14 & $1 \cdot 1$ & 13 & 10 & $1 \cdot 3$ & 6 & 7 & 0.9 & 5 & 11 & 0.4 & 25 & 15 & 1.5 & 3 & 2 & 1.4 & 1 & 2 & 0.4 \\
\hline Embryonal CNS & 21 & 9 & $4 \cdot 2$ & 14 & 9 & $2 \cdot 5$ & 6 & 3 & $3 \cdot 1$ & 9 & 3 & $6 \cdot 3$ & 0 & & & & 2 & $4 \cdot 2$ & 2 & 1 & $5 \cdot 2$ \\
\hline Neuroblastoma & 15 & 4 & 5.7 & 8 & 5 & $1 \cdot 7$ & 1 & 1 & $2 \cdot 1$ & 7 & 2 & $5 \cdot 4$ & 12 & 4 & $6 \cdot 2$ & 1 & 2 & $1 \cdot 3$ & 1 & 1 & 1.8 \\
\hline Retinoblastoma & 10 & 1 & $14 \cdot 0$ & 3 & 5 & 0.5 & 3 & 2 & 1.5 & 3 & 2 & 1.8 & 22 & 1 & $30 \cdot 7$ & 1 & 1 & $1 \cdot 1$ & 1 & 2 & 0.5 \\
\hline Trachea & 10 & 18 & 0.9 & 5 & 4 & $1 \cdot 1$ & 4 & 5 & 0.9 & 2 & 4 & 0.4 & 11 & 11 & $1 \cdot 1$ & 3 & 1 & 3.8 & 1 & 1 & 0.5 \\
\hline
\end{tabular}

need for strong political commitment to ensure centralisation, to make sure all patients with rare cancer receive the highest quality of care.

Cancer care was highly centralised in Slovenia. Additionally, the major hospitals were organised on a task-specific basis: radiotherapy was only provided by the National Cancer Center, whereas surgical treatment was more often done in two other major hospitals. Reducing delays in diagnosis and treatment was recognised in Slovenia as one of the major challenges to improve rare cancer outcomes.

Navarra is a relatively small region of Spain, a country with a highly regionalised health organisation. No hospital with national recruitment for rare cancers was operating in Navarra, and $98 \%$ of resident patients with rare cancer were treated locally, the majority in the two largest regional hospitals. However, the admission volumes of Navarra hospitals were much lower than in all the other participating countries, even considering some underestimation due to unregistered patients coming from outside the region. This suggests some disadvantages in organising rare cancer treatment on a regional or local basis.

To conclude, to our knowledge, this is the largest study that estimates the burden of rare cancer for Europe, including trends in incidence and survival rates. This study also provides indicators of rare cancer treatment management. In seven European countries we observed, with few exceptions, a low level of centralisation of treatment for rare cancers. We recognise the importance of population-based cancer registries in descriptive studies like this, to ensure surveillance. However, the quality of the data needs to be improved when morphology, hospital, and treatment definitions are considered. To this aim, we suggest the use of specific 
data quality indicators, the planning of periodic samplebased quality studies and, above all, a wider use of these variables in population-based studies, with related sensitivity analysis. Furthermore, the international classification for cancer have to rapidly include the new entities on the basis of molecular and genomic categorisation. Genomic categorisation is a necessary condition for updating a new rare cancers list.

The European Network of Cancer Registries (ENCR) should work to boost these quality improvements and make wider use of the data on rare cancers. The Joint Action of Rare Cancers ${ }^{28}$ and the European Network for Rare Diseases will profit from these data, which are also useful for national and European policies to improve care for patients with rare cancer. The RARECAREnet project website includes a search tool with data for all the countries that contributed data.

\section{Contributions}

GG, RC, and AT designed the study and wrote the article. RC, LB, SM, and RDA did the statistical analyses. LB, RDA, SM, EA, HC, ND, MKL, SS, JMvdZ, LVE, OV, MPZ, LAA, FB, IK, RO, and CAS revised the paper and contributed to data interpretation. All authors reviewed and approved the final version. Members of the working group collected data.

\section{Declaration of interests}

We declare no competing interests.

\section{Acknowledgments}

We thank J D Baggott for editing, and L Buratti for technical assistance (Evaluative Epidemiology Unit, Fondazione IRCCS Istituto Nazionale Tumori, Milan, Italy). This research was funded by the European Commission through the Consumers, Health, Agriculture and Food Executive Agency (Chafea); Grant No. 2000111201; lnformation network on rare cancers"-RARECARENet.

\section{References}

1 Gatta G, van der Zwan JM, Casali PG, et al. Rare cancers are not so rare: the rare cancer burden in Europe. Eur J Cancer 2011; 47: $2493-511$.

2 Casali PG. Improving methodology to go beyond histology in rare cancers. Lancet Oncol 2013; 14: 276-77.

3 RARECARENet. Information Network on Rare Cancers. http://www.rarecarenet.eu/rarecarenet/ (accessed March 1, 2017).

4 Rossi S, Baili P, Capocaccia R, et al. The EUROCARE-5 study on cancer survival in Europe 1999-2007: database, quality checks and statistical analysis methods. Eur J Cancer 2015; published online Sept 6. DOI:10.1016/j.ejca.2015.08.001.

5 RARECARENet. Information Network on Rare Cancers. Indicators. http://www.rarecarenet.eu/rarecarenet/index.php/indicators (accessed March 1, 2017).

6 Ederer F, Axtell LM, Cutler SJ. The relative survival: a statistical methodology. Natl Cancer Inst Monogr 1961; 6: 101-21.

7 Quaresma M, Coleman MP, Rachet B. Funnel plots for population-based cancer survival: principles, methods and applications. Stat Med 2014; 33: 1070-80.

8 De Angelis R, Sant M, Coleman MP, et al. Cancer survival in Europe 1999-2007 by country and age: results of EUROCARE-5a population-based study. Lancet Oncol 2014; 15: 23-34.

9 Percy C, Fritz A, Jack A, et al. International classification of diseases for oncology (ICD-O). 3rd ed. Geneva: World Health Organisation, 2000 .
10 Trama A, Marcos-Gragera R, Sánchez Pérez MJ, et al. Data quality in rare cancers registration: the report of the RARECARE data quality study. Tumori 2017; 103: 22-32.

11 HAEMACARE Working Group. Manual for coding and reporting haematological malignancies. Tumori 2010; 96: i-A32.

12 Vaccarella S, Franceschi S, Bray F, Wild CP, Plummer M, Dal Maso L. worldwide thyroid-cancer epidemic? The increasing impact of overdiagnosis. N Engl J Med 2016; 375: 614-17.

13 Curado MP, Hashibe M. Recent changes in the epidemiology of head and neck cancer. Curr Opin Oncol 2009; 21: 194-200.

14 Heck JE, Berthiller J, Vaccarella S, et al. Sexual behaviours and the risk of head and neck cancers: a pooled analysis in the International Head and Neck Cancer Epidemiology (INHANCE) consortium. Int J Epidemiol 2010; 39: 166-81.

15 Gavin AT, Francisci S, Foschi R, et al. Oesophageal cancer survival in Europe: a EUROCARE-4 study. Cancer Epidemiol 2012; 36: 505-12.

16 WHO. The European Tobacco Control Report 2007. http://www.euro.who.int/_data/assets/pdf_file/0005/68117/ E89842.pdf (accessed April 15, 2017).

17 Kantarjian H, O’Brien S, Jabbour E, et al. Improved survival in chronic myeloid leukemia since the introduction of imatinib therapy: a single institution historical experience. Blood 2012; 119: 1981-87.

18 Sant M, Minicozzi P, Mounier M, et al. Survival for haematological malignancies in Europe between 1997 and 2008 by region and age: results of EUROCARE-5, a population-based study. Lancet Oncol 2014; 15: 931-42.

19 Lepage C, Bouvier AM, Faivre J. Endocrine tumours: epidemiology of malignant digestive neuroendocrine tumours. Eur J Endocrinol 2013; 168: R77-83.

20 Lepage C, Capocaccia R, Hackl M, et al. Survival in patients with primary liver cancer, gallbladder and extrahepatic biliary tract cancer and pancreatic cancer in Europe 1999-2007: results of EUROCARE-5. Eur J Cancer 2015; 51: 2169-78.

21 O'Rorke MA, Ellison MV, Murray LJ, Moran M, James J, Anderson LA. Human papillomavirus related head and neck cancer survival: a systematic review and meta-analysis. Oral Oncol 2012; 48: 1191-201.

22 Trama A, Foschi R, Larrañaga N, et al. Survival of male genital cancers (prostate, testis and penis) in Europe 1999-2007: results from the EUROCARE-5 study. Eur J Cancer 2015; 51: 2206-16.

23 Botta L, Trama A, Capocaccia R, Gatta G, Pilot Study WG. Hospital volume analysis for head and neck cancers: results from the RARECARENet study. 40 Reunìo del Group per l'Epidemiologia i l'Enregistrament del Càncer en els Paosos de Llengua Llatina. GRELL 13-15 Maig 2015 Resus. https://drive.google.com/file/d/ 0B450I5xTZUGjNVFFbExmeWlrd1U/view (accessed March 10, 2017).

24 Weitz J, Koch M, Friess H, Büchler MW. Impact of volume and specialization for cancer surgery. Dig Surg 2004; 21: 253-61.

25 van Harten MC, de Ridder M, Hamming-Vrieze O, Smeele LE, Balm AJ, van den Brekel MW. The association of treatment delay and prognosis in head and neck squamous cell carcinoma (HNSCC) patients in a Dutch comprehensive cancer center. Oral Oncol 2014; 50: 282-90.

26 Shin DW, Cho J, Yang HK, et al. Oncologist Perspectives on Rare Cancer Care: A Nationwide Survey. Cancer Res Treat 2015; 47: 591-99.

27 Joint Action on Rare Cancers Launch. European Cancer Patient Coalition. http://ecpc.org/Documents/Projects/RARECAREnet (accessed March 10, 2017).

28 Rodwell C, Aymé S. 2014 Report on the State of the Art of Rare Disease Activities in Europe. July, 2014. http://ec.europa.eu/ transparency/regdoc/rep/3/2014/EN/3-2014-1408-EN-F1-1.Pdf (accessed March 10, 2017). 